\title{
MARCO DEGIOVANNi
}

\section{Bifurcation problems for nonlinear elliptic variational inequalities}

\author{
Annales de la faculté des sciences de Toulouse $5^{e}$ série, tome 10, \\ $\mathrm{n}^{\mathrm{o}} 2$ (1989), p. 215-258 \\ <http://www.numdam.org/item?id=AFST_1989_5_10_2_215_0>
}

(C) Université Paul Sabatier, 1989, tous droits réservés.

L'accès aux archives de la revue «Annales de la faculté des sciences de Toulouse » (http://picard.ups-tlse.fr/ annales/) implique l'accord avec les conditions générales d'utilisation (http://www.numdam.org/conditions). Toute utilisation commerciale ou impression systématique est constitutive d'une infraction pénale. Toute copie ou impression de ce fichier doit contenir la présente mention de copyright.

\section{NumDam}

Article numérisé dans le cadre du programme Numérisation de documents anciens mathématiques http://www.numdam.org/ 


\title{
Bifurcation problems for nonlinear elliptic variational inequalities
}

\author{
Marco Degiovanni ${ }^{(1)}$
}

REsumé.- On étudie un problème de bifurcation variationnelle associé à des fonctionnelles non régulières. On prouve un théorème général de bifurcation de la première valeur propre. On donne aussi un résultat de bifurcation pour les valeurs propres successives et un résultat de multiplicité. On montre une application aux inéquations variationnelles elliptiques du second ordre.

Abstract. - A problem of variational bifurcation associated with nonsmooth functionals is studied. A general bifurcation theorem for the first eigenvalue is proved. Also the bifurcation from higher eigenvalues and a multiplicity result are given. An application to second order elliptic variational inequalities is shown.

\section{Introduction}

Eigenvalue problems for nonlinear elliptic variational inequalities of the form

$$
\left\{\begin{array}{l}
(\lambda, u) \in \mathbf{R} \times \mathbf{K} \\
\langle A(u), v-u\rangle \geq \lambda<L u, v-u\rangle \quad \forall v \in \mathbf{K}
\end{array}\right.
$$

where $\mathbf{K}$ is a convex subset of some functional space, $A$ a nonlinear potential operator and $L$ a symmetric linear operator, have been considered by several authors (see $[3,4,8,9,19,20,23,24,25,29,30,31,32,33,36,37,40,41$, 42]).

Some of them $([19,20,24,29,30,31,32,33,37,42])$ treat the bifurcation problem, which is the study, under the further assumptions that $0 \in \mathbf{K}$ and

(1) Facoltà di Ingegneria, Università di Brescia, Via Valotti 9, 25060 Brescia, Italy

This work was partially supported by a national research grant financed by the Ministero della Pubblica Istruzione (40\%-1985). 
$A(0)=0$, of the real numbers $\lambda_{0}$ such that the pair $\left(\lambda_{0}, 0\right)$ accumulates solutions $(\lambda, u)$ of (1.1) with $u \neq 0$.

As in the case of equations [22], it is true under reasonable assumptions that every $\lambda_{0}$ of bifurcation for (1.1) is an eigenvalue of the "linearized" problem

$$
\left\{\begin{array}{l}
(\lambda, u) \in \mathbf{R} \times \mathbf{K}_{\mathbf{0}} \\
<A^{\prime}(0) u, v-u>\geq \lambda<L u, v-u>\quad \forall v \in \mathbf{K}_{0}
\end{array}\right.
$$

namely $\left(\lambda_{0}, u\right)$ satisfies (1.2) for some $u \neq 0$, where $\mathbf{K}_{0}$ is the convex cone defined as the closure of $\bigcup_{t>0} t \mathbf{K}$.

About the converse, which is also true for equations [22], only partial results are available.

The problem has mainly two difficulties. If we use the variational approach, which yields the sharpest results in the case of equations, we have to apply critical point theory to the potential of $A$ constrained on the sets $\mathbf{K} \cap\left\{u: \frac{1}{2}(L u \mid u)= \pm \rho^{2}\right\}$, which are not smooth. A further difficulty comes from the fact that the convex set $\mathbf{K}$ and the manifolds $\left\{u: \frac{1}{2}(L u \mid u)= \pm \rho^{2}\right\}$ may be "tangent" in a sense that has been precised in $[8,9]$.

The first difficulty can be overcome by considering only the (at most) two eigenvalues of (1.2) which correspond to minimize $\left\{u \mapsto \frac{1}{2}\left(A^{\prime}(0) u \mid u\right)\right\}$ on the sets $K_{0} \cap\left\{u: \frac{1}{2}(L u \mid u)= \pm 1\right\}$, as it is done in $[19,20,29,37]$. The second one by assuming that $\mathbf{K}$ is a convex cone. Infact, if $\mathbf{K}$ is a convex cone, $\mathbf{K}$ and $\left\{u: \frac{1}{2}(L u \mid u)= \pm \rho^{2}\right\}$ are never tangent. Actually, in [19, 20, $24,30,31,32,33,37,42$ ] the set $\mathbf{K}$ is supposed to be a convex one. In [29] this condition is not required, but an extra assumption is imposed in order to avoid tangency.

Our first purpose is to give a general bifurcation result (Theorems 3.21 and 4.14) for the eigenvalues of (1.2) corresponding to minimize $\left\{u \mapsto \frac{1}{2}\left(A^{\prime}(0) u \mid u\right)\right\}$ on $\mathrm{K} \cap\left\{u \mapsto \frac{1}{2}(L u \mid u)= \pm 1\right\}$. The crucial point is the following : if $\mathcal{A}$ is a potential of $A, \rho_{h} \rightarrow 0$ and $\mathbf{K}$ and $\left\{u: \frac{1}{2}(L u \mid u)= \pm \rho_{h}^{2}\right\}$ are tangent at some $u_{h}$, then $\mathcal{A}\left(u_{h}\right) / \rho_{h}^{2} \rightarrow+\infty$. Therefore the possible tangency points does not interact with the minimization technique. Then 
it is possible to apply the generalized theorems on Lagrange multipliers contained in $[8,9]$.

Another purpose is to provide a bifurcation result for all eigenvalues of (1.2) under the assumption that $\mathbf{K}_{0}$ is a linear space (Theorems 3.29 and 4.16). In this case the main tool is contituted by the techniques of critical point theory for nonsmooth functions developed in [11, 13, 18, 27]. These last results are obtained as particular cases of more general theorems (3.20 and 4.13) involving a topological assumption on the eigenvalue of (1.2) under consideration.

In the case in which $\mathbf{K}_{\mathbf{0}}$ is under a linear space, also a multiplicity result is given (Theorems 3.30 and 4.18) for simple eigenvalues.

In section 3 we develop an abstract theory of variational bifurcation for nonsmooth functions. The main results were announced in $[15,16,17]$ in the case in which $L$ is the identity map.

In section 4 we show an application to some nonlinear elliptic variational inequalities of second order (in which $L$ is the identity map). An application to elasticity, in which $L$ is not the identity map, is contained in [14].

\section{Some recalls of nonsmooth analysis}

In this section we recall some notions and results of nonsmooth analysis $[8,9,11,13,18,27]$ which will be used later. For the elementary notions of homotopy theory involved here, the reader is referred to [38].

Throughout this section $H$ will denote a real Hilbert space. The scalar product, norm and metric of $H$ will be denoter by $(\cdot \mid \cdot),|\cdot|$ and $d_{H}$ respectively, while $B(u, r)$ will denote the open ball of center $u$ and radius $r$.

Let $W$ be an open subset of $H$ and

$$
f: W \longrightarrow \mathbf{R} \cup\{+\infty\}
$$

a function. We set

$$
\begin{gathered}
D(f)=\{u \in W: f(u)<+\infty\} \\
\forall c \in \mathbf{R} \cup\{+\infty\}, \quad f^{c}=\{u \in D(f): f(u) \leq c\} .
\end{gathered}
$$

For every $u$ in $D(f)$ let us denote by $\partial^{-} f(u)$ the (possibly empty) set of $\alpha^{\prime} s$ in $H$ such that

$$
\begin{gathered}
\liminf _{v \rightarrow u} \frac{f(v)-f(u)-(\alpha \mid v-u)}{|v-u|} \geq 0 . \\
-217-
\end{gathered}
$$


We set also $\partial^{-} f(u)=\emptyset, \forall u \in W \backslash D(f)$ and

$$
D\left(\partial^{-} f\right)=\left\{u \in W: \partial^{-} f(u) \neq \emptyset\right\} .
$$

Since $\partial^{-} f(u)$ is convex and closed, for every $u$ in $D\left(\partial^{-} f\right)$ we can denote by $\operatorname{grad}^{-} f(u)$ the element of $\partial^{-} f(u)$ having minimal norm.

If $W=H$ and $f$ is convex, the notion of $\partial^{-} f$ coincides with the usual notion of subdifferential in convex analysis. If $g: W \rightarrow \mathbf{R}$ is Fréchet differentiable at $u \in W$, then $\partial^{-}(f+g)(u)=\partial^{-} f(u)+\operatorname{grad} g(u)$.

Definition 2.1. - A point $u \in W$ is said to be critical from below for $f$, if $0 \in \partial^{-} f(u)$. A real number $c$ is said to be critical from below for $f$, if there exists $u \in W$ such that $0 \in \partial^{-} f(u), f(u)=c$.

DEFINITION 2.2. - The function $f$ is said to have a $\varphi$-monotone subdifferential of order two, if there exists a continuous function $\mathcal{X}:(D(f))^{2} \times$ $\mathbf{R}^{2} \rightarrow \mathbf{R}^{+}$such that

$$
(\alpha-\beta \mid u-v) \geq-\mathcal{X}(u, v, f(u), f(v))\left(1+|\alpha|^{2}+|\beta|^{2}\right)|u-v|^{2}
$$

whenever $u, v \in D\left(\partial^{-} f\right), \alpha \in \partial^{-} f(u), \beta \in \partial^{-} f(v)$.

Definition 2.3. - Let $c$ be a real number. The function $f$ is said to verify the Palais-Smale condition at level c (or, briefly, $\left.(P S)_{c}\right)$, if for every sequence $\left(u_{h}\right)$ in $D\left(\partial^{-} f\right)$ with $\lim _{h} \operatorname{grad}^{-} f\left(u_{h}\right)=0, \lim _{h} f\left(u_{h}\right)=c$, there exists a subsequence $\left(u_{h_{k}}\right)$ converging to an element of $W$.

Remark 2.4. - Let us assume that $f$ is lower semicontinuous and has a $\varphi$-monotone subdifferential of order two. Then the set

$\left\{c \in \mathbf{R}: c\right.$ is not critical from below for $f$ and $(P S)_{c}$ holds $\}$ is open in $\mathbf{R}$.

Proof.-See [13, Remark 4.2].

Besides the metric $d_{H}$ induced by $H$, it is convenient to consider on $D(f)$ also the graph metric $d^{*}$ defined by

$$
d^{*}(u, v)=|u-v|+|f(u)-f(v)| .
$$

However, when the metric is not specified, we mean that $D(f)$ is endowed with the metric $d_{H}$.

TIIEOREM 2.6. - Let us suppose that $f$ is lower semicontinuous and has $a \varphi$-monotone subdifferential of order two. Let $-\infty<a \leq b<+\infty$. 
Bifurcation problems for nonlinear elliptic variational inequalities

Then the pair $\left(f^{b}, f^{a}\right)$ endowed with the metric $d^{*}$ is homotopically equivalent to the pair $\left(f^{b}, f^{a}\right)$ endowed with the metric $d_{H}$. In particular, $f^{a}$ is a weak deformation retract of $f^{b}$ with respect to the metric $d^{*}$ if and only if the same fact occurs in the metric $d_{H}$.

Proof.-See [13, Theorem 3.18].

As in classical critical point theory, also in the nonsmooth case an important tool is constituted by the deformation lemma (see [27, Lemma 3.10]). Here we recall a version taken from [13].

LEMMA 2.7. - Let us suppose that $f$ is lower semicontinuous and has a $\varphi$-monotone subdifferential of order two. Let $-\infty<a \leq b<+\infty$. Let us assume that

i) for every $c$ in $[a, b], c$ is not critical from below for $f$;

ii) for every $c$ in $\left[a, b\left[, f^{c}\right.\right.$ is closed in $H$;

iii) for every $c$ in $\left[a, b\left[\right.\right.$, the function $f$ verifies $(P S)_{c}$.

Then $f^{a}$ is a weak deformation retract of $f^{b}$.

Proof. - If we consider the metric $d^{*}$, we result is contained in [19, Lemma 4.4]. For the metric $d_{H}$ we can apply Theorem 2.6.

DEFINITION 2.8. - A real number $c$ is said to be essential for $f$, if there exists two sequences $\left(a_{k}\right)$ in $]-\infty, c\left[\right.$ and $\left(b_{k}\right)$ in $] c,+\infty[$ converging to $c$ such that:

i) $\forall k \in \mathbf{N}, a_{k}$ and $b_{k}$ are not critical from below for $f$;

ii) $\forall k \in \mathbf{N}$, the set $f^{a_{k}}$ is not a weak deformation retract of $f^{b_{k}}$, endowed with the metric $d^{*}$.

If $A$ is a subset of $H$, we define a function $I_{A}: H \rightarrow \mathbf{R} \cup\{+\infty\}$ by

$$
I_{A}(u)=\left\{\begin{array}{ll}
0 & u \in A \\
+\infty & u \in H \backslash A
\end{array} .\right.
$$

For every $u$ in $A, \partial^{-} I_{A}(u)$ is a closed convex cone (in some sense, the outward normal cone to $A$ at $u$ ).

Remark 2.9. - If $M$ is a hypersurface in $H$ of class $C^{1}$, we have for every $u$ in $M$

$$
\begin{aligned}
\partial^{-} I_{M}(u)= & \{\lambda v(u): \lambda \in \mathbf{R}\} \\
& -219-
\end{aligned}
$$


where $v(u)$ is a normal unit vector to $M$ at $u$.

Definition 2.10.- Let $A$ and $B$ be two subsets of $H$ and $u \in A \cap B$. Then $A$ and $B$ are said to be (outwardly) tangent at $u$, if

$$
\partial^{-} I_{A}(u) \cap-\left(\partial^{-} I_{B}(u)\right) \neq\{0\}
$$

Remark 2.11. - Let $A$ be a subset of $H, C$ a convex subset of $H$ and $u \in A \cap C$. Then $A$ and $C$ are not tangent at $u$ if and only if

$$
\forall v \in \partial^{-} I_{A}(u) \backslash\{0\}, \exists w \in C:(v \mid w-u)<0 .
$$

THEOREM 2.12. - Let $M$ be a hypersurface in $W$ of class $C^{1}$ and

$$
f: W \rightarrow \mathbf{R} \cup\{+\infty\}
$$

a lower semicontinuous function such that, for some continuous function $q: D(f) \rightarrow \mathbf{R}$,

$$
f(v) \geq f(u)+(\alpha \mid v-u)-q(u)|v-u|^{2}
$$

whenever $c \in W, u \in D\left(\partial^{-} f\right), \alpha \in \partial^{-} f(u)$.

Let $u_{0} \in D(f) \cap M$ and let us assume that $D(f)$ and $M$ are not tangent at $u_{0}$.

Then we have

$$
\partial^{-}\left(f+I_{M}\right)\left(u_{0}\right)=\partial^{-} f\left(u_{0}\right)+\partial^{-} I_{M}\left(u_{0}\right) .
$$

Proof. - See [8, Theorem 1.13 and Remark $\left.1.12_{b}\right]$.

Finally, let us recall the notion of $\Gamma$-convergence (epiconvergence in the language of [1]) from [12].

DEFINITION 2.13. - Let $X$ be a topological space and

$$
g_{h}: X \rightarrow \mathbf{R} \cup\{+\infty\} \quad(h \in \overline{\mathbf{N}}:=\mathbf{N} \cup\{\infty\})
$$

a sequence of functions. We say that

$$
\begin{aligned}
g_{\infty}= & \Gamma^{-}(X) \lim _{h} g_{h} \\
& -220-
\end{aligned}
$$


Bifurcation problems for nonlinear elliptic variational inequalities

is the following facts hold:

i) for every $u$ in $X$, for every sequence $\left(u_{h}\right)$ in $X$ converging to $u$, we have

$$
g_{\infty}(u) \leq \liminf _{h} g_{h}\left(u_{h}\right)
$$

ii) for every $u$ in $X$ there exists a sequence $\left(u_{h}\right)$ in $X$ converging to $u$ such that

$$
g_{\infty}(u)=\lim _{h} g_{h}\left(u_{h}\right)
$$

From now on in this section we shall consider a sequence of functions

$$
f_{h}: H \rightarrow \mathbf{R} \cup\{+\infty\} \quad(h \in \overline{\mathbf{N}})
$$

DEFINITION 2.14. - The sequence $\left(f_{h}\right)$ is said to be equicoercive, if for every real number $c$ the closure of the set

$$
\bigcup_{h \in \mathbb{N}}\left\{u \in H: f_{h}(u) \leq c\right\}
$$

is compact.

Remark 2.15. - Let us suppose that $\left(f_{h}\right)$ is equicoercive and that $f_{\infty}=\Gamma^{-}(H) \lim _{h} f_{h}$. Then for every real number $c$ the set $f_{\infty}^{c}$ is compact. Therefore the closure of the set $\underset{h \in \bar{N}}{U} f_{h}^{c}$ is compact.

THEOREM 2.16. - Let us suppose that

i) for every $h$ in $\overline{\mathrm{N}}, f_{h}$ is lower semicontinuous;

ii) there exists a continuous function

$$
\chi:\left(\bigcup_{h \in \bar{N}} D\left(f_{h}\right)\right)^{2} \times \mathbf{R}^{2} \rightarrow \mathbf{R}^{+}
$$

such that

$$
\begin{gathered}
(\alpha-\beta \mid u-v) \geq \\
\geq-\chi\left(u, v, f_{h}(u), f_{h}(v)\right)\left(1+|\alpha|^{2}+|\beta|^{2}\right)|u-v|^{2}
\end{gathered}
$$

whenever $h \in \overline{\mathbf{N}}, u, v \in D\left(\partial^{-} f_{h}\right), \alpha \in \partial^{-} f_{h}(u), \beta \in \partial^{-} f_{h}(v)$; 
iii)

$$
f_{\infty}=\Gamma^{-}(H) \lim _{h} f_{h}
$$

iv) the sequence $\left(f_{h}\right)$ is equicoercive.

Let $-\infty<a \leq b<+\infty$ and let us assume that $a$ and $b$ are not critical from below for $f_{\infty}$.

Then there exists $h_{0} \in \mathbf{N}$ such that for every $h \geq h_{0}$ the pair $\left(f_{h}^{b}, f_{h}^{a}\right)$ is homotopically equivalent to the pair $\left(f_{\infty}^{b}, f_{\infty}^{a}\right)$.

Proof.-By Remark 2.15 we can apply [13, Theorem 5.12 and Remark 5.13].

We conclude this section by proving a result which will play an essential role in the study of bifurcation.

THeOREM 2.17. - Let $M$ be a hypersurface in $H$ of class $C_{\mathrm{loc}}^{1,1}$.

Let us suppose that

a) $M$ is a closed subset of $H$ and for every $h$ in $\overline{\mathbf{N}}, f_{h}$ is lower semicontinuous ;

b) there exists $q$ in $\mathbf{R}^{+}$such that for every $h$ in $\overline{\mathbf{N}}$ the function $\left\{u \mapsto f_{h}(u)+q|u|^{2}\right\}$ is convex;

c)

$$
f_{\infty}=\Gamma^{-}(H) \lim _{h} f_{h}
$$

d) the sequence $\left(f_{h}+I_{M}\right)$ is equicoercive;

e) for every $u$ in $D\left(f_{\infty}\right) \cap M, D\left(f_{\infty}\right)$ and $M$ are not tangent at $u$.

Then there exists a sequence $\left(\widehat{f}_{h}\right)$ of functions $\widehat{f}_{h}: H \rightarrow \mathbf{R} \cup\{+\infty\}$ $(h \in \overline{\mathbf{N}})$ with the following properties :

i) $\widehat{f}_{\infty}=f_{\infty} ; \forall h \in \mathbf{N}, \widehat{f}_{h} \geq f_{h}$;

ii) $\forall h \in \mathbf{N}, \forall u, v \in H, f_{h}(u)=f_{h}(v)$ and $|u|=|v|$ imply $\widehat{f}_{h}(u)=\widehat{f}_{h}(v)$;

iii) $\forall h \in \mathbf{N}, \widehat{f}_{h}$ is lower semicontinuous;

iv) $\forall h \in \mathbf{N}$, the function $\left\{u \mapsto \widehat{f}_{h}(u)+q|u|^{2}\right\}$ is convex;

v)

$$
\widehat{f}_{\infty}=\Gamma^{-}(H) \lim _{h} \widehat{f}_{h} \text {; }
$$

vi) $\forall h \in \mathbf{N}, \forall u \in D\left(\widehat{f}_{h}\right) \cap M, D\left(\widehat{f_{h}}\right)$ and $M$ are not tangent at $u$;

iv) if $\left(u_{h}\right)$ is a sequence in $M$ with $\lim s u p \widehat{f}_{h}\left(u_{h}\right)<+\infty$, we have $h$

$$
\widehat{f}_{h}\left(u_{h}\right)=f_{h}\left(u_{h}\right), \partial^{-} \widehat{f}_{h}\left(u_{h}\right)=\partial^{-} f_{h}\left(u_{h}\right)
$$

eventually as $h \rightarrow+\infty$. 
Bifurcation problems for nonlinear elliptic variational inequalities

Moreover, if we set $\tilde{f}_{h}=\widehat{f}_{h}+I_{M} \forall h \in \mathbf{N}$, the following facts hold :

viii) $\forall h \in \mathbf{N}, \widetilde{f}_{h}$ is lower semicontinuous;

ix) there exists a continuous function

$$
\varphi_{0}:\left(\bigcup_{h \in \overline{\mathbf{N}}} D\left(\tilde{f}_{h}\right)\right) \times \mathbf{R} \rightarrow \mathbf{R}^{+}
$$

such that

$$
\tilde{f}_{h}(v) \geq \tilde{f}_{h}(u)+(\alpha \mid v-u)-\varphi_{0}\left(u, \tilde{f}_{h}(u)\right)(1+|\alpha|)|v-u|^{2}
$$

whenever $h \in \mathbf{N}, v \in H, u \in D\left(\partial^{-} \widetilde{f}_{h}\right), \alpha \in \partial^{-} \widetilde{f}_{h}(u)$;

in particular, there exists a continuous function

$$
\mathcal{X}:\left(\bigcup_{h \in \overline{\mathbf{N}}} D\left(\tilde{f}_{h}\right)\right)^{2} \times \mathbf{R}^{2} \rightarrow \mathbf{R}^{+}
$$

such that

$$
\begin{gathered}
(\alpha-\beta \mid u-v) \geq \\
\geq-\mathcal{X}\left(u, v, \tilde{f}_{h}(u), \tilde{f}_{h}(v)\right)\left(1+|\alpha|^{2}+|\beta|^{2}\right)|u-v|^{2}
\end{gathered}
$$

whenever $h \in \mathbf{N}, u, v \in D\left(\partial^{-} \widetilde{f}_{h}\right), \alpha \in \partial^{-} \tilde{f}_{h}(u), \beta \in \partial^{-} \widetilde{f}_{h}(v)$;

x) $\quad \widetilde{f}_{\infty}=\Gamma^{-}(H) \lim _{h} \widetilde{f}_{h}$;

xi) the sequence $\left(\tilde{f}_{h}\right)$ is equicoercive.

Proof. - For every $h \in \mathbf{N}$, we set

$c_{h}=\inf \left\{f_{h}(u): u \in D\left(f_{h}\right) \cap M, D\left(f_{h}\right)\right.$ and $M$ are tangent at $\left.u\right\}$ with the convention $\inf \emptyset=+\infty$.

First of all we claim that

$$
\lim _{h} c_{h}=+\infty .
$$

Indeed, by contradiction, we could find a subsequence $\left(f_{h_{k}}\right)$ and a sequence $\left(u_{k}\right)$ in $H$ such that $u_{k} \in D\left(f_{h_{k}}\right) \cap M, D\left(f_{h_{k}}\right)$ and $M$ are tangent at $u_{k}$ and $\sup _{k} f_{h_{k}}\left(u_{k}\right)<+\infty$. 
By equicoercivity, up to a subsequence $\left(u_{k}\right)$ converges to some $u$ in $M$. Moreover by $\Gamma$-convergence $u \in D\left(f_{\infty}\right)$. By Remark 2.11

$$
\forall k \in \mathbf{N}, \exists v_{k} \in \partial^{-} I_{M}\left(u_{k}\right) \backslash\{0\}: \forall w \in D\left(f_{h_{k}}\right),\left(v_{k} \mid w-u_{k}\right) \geq 0 .
$$

We can suppose that $\left|v_{k}\right|=1$. Because of Remark 2.9 , up to a subsequence $\left(v_{k}\right)$ converges to some $v \in \partial^{-} I_{M}(u)$ with $|v|=1$. Again by Remark 2.11 and assumption e) there exists $w \in D\left(f_{\infty}\right)$ such that $(v \mid w-u)<0$. Let $\left(w_{k}\right)$ be a sequence converging to $w$ with $\lim _{k} f_{h_{k}}\left(w_{k}\right)=f_{\infty}(w)$. Then we have

$$
w_{k} \in D\left(f_{h_{k}}\right),\left(v_{k} \mid w_{k}-u_{k}\right)<0
$$

eventually as $k \rightarrow+\infty$, which is a contradiction.

Now for every $h \in \mathbf{N}$, let $\vartheta_{h}: \mathbf{R} \rightarrow \mathbf{R} \cup\{+\infty\}$ be an increasing lower semicontinuous convex function such that

$$
\begin{gathered}
\forall x \leq c_{h}-1, \quad \vartheta_{h}(x)=x ; \\
\forall x \in] c_{h}-1, c_{h}\left[, \quad x \leq \vartheta_{h}(x)<+\infty\right. \\
\forall x \geq c_{h}, \quad \vartheta_{h}(x)=+\infty
\end{gathered}
$$

We set $\widehat{f}_{\infty}=f_{\infty}$ and $\forall h \in \mathbf{N}$,

$$
\widehat{f}_{h}(u)=\left\{\begin{array}{ll}
\vartheta_{h}\left(f_{h}(u)+q|u|^{2}\right)-q|u|^{2} & u \in D\left(f_{h}\right) \\
+\infty & u \in H \backslash D\left(f_{h}\right)
\end{array} .\right.
$$

Then properties i), ii), iii), iv and v) are immediate.

Let us prove vi). Let $h \in \mathbf{N}, u \in D\left(\widehat{f}_{h}\right) \cap M$ and $v \in \partial^{-} I_{M}(u) \backslash\{0\}$. Since $f_{h}(u)<c_{h}-q|u|^{2} \leq c_{h}$, there exists $w \in D\left(f_{h}\right)$ with $(v \mid w-u)<0$. Then, if $t \in] 0,1]$ is sufficiently small, we have $(u+t(w-u)) \in D\left(\hat{f}_{h}\right)$ and $\left(v \mid u+t(w-u)-u<0\right.$. By Remark 2.11, D( $\left.\widehat{f}_{h}\right)$ and $M$ are not tangent at $u$.

Let us prove vii). Since $\widehat{f}_{h}=f_{h}$, by assumption d) we have that, up to a subsequence, $\left(u_{h}\right)$ converges to some $u$ in $M$. Then it is obvious that $\widehat{f}_{h}\left(u_{h}\right)=f_{h}\left(u_{h}\right)$ eventually as $h \rightarrow+\infty$. Since $\widehat{f}_{h} \geq f_{h}$, we have $\partial^{-} f_{h}\left(u_{h}\right) \subset \partial^{-} \widehat{f}_{h}\left(u_{h}\right)$ eventually as $h \rightarrow+\infty$. On the other hand for large $h$

$$
f_{h}(v)+q|v|^{2} \leq c_{h}-1
$$

whenever $v \in B\left(u_{h}, 1\right)$ and $f_{h}(v)<f_{h}\left(u_{h}\right)+1$. Therefore, if $h$ is sufficiently large, we have $\widehat{f}_{h}(v)=f_{h}(v)$ whenever $v \in B\left(u_{h}, 1\right)$ and $f_{h}(v)<f_{h}\left(u_{h}\right)+1$. In particular $\partial^{-} \hat{f}_{h}\left(u_{h}\right) \subset \partial^{-} f_{h}\left(u_{h}\right)$. 
Bifurcation problems for nonlinear elliptic variational inequalities

Now we set $\forall h \in \overline{\mathbf{N}}, \widetilde{f}_{h}=\widehat{f}_{h}+I_{m}$.

Property viii) is obvious. Since $\widetilde{f}_{h} \geq f_{h}+I_{M^{\prime}} x i$ ) follows from d).

Let us remark that by iv) we have

$$
\widehat{f}_{h}(v) \geq \widehat{f}_{h}(u)+(\alpha \mid v-u)-q|v-u|^{2}
$$

whenever $h \in \overline{\mathbf{N}}, u \in D\left(\partial^{-} \widehat{f}_{h}\right), \alpha \in \partial^{-} \widehat{f}_{h}(u)$.

Let us prove $\mathrm{x}$ ). Since $M$ is closed, by $\mathrm{v}$ ) we have only to prove that $\forall u \in H, \exists\left(u_{h}\right)$ converging to $u$ with $\lim _{h} \tilde{f}_{h}\left(u_{h}\right)=\tilde{f}_{\infty}(u)$. Actually it is sufficient to show that $\forall u \in D\left(\widehat{f}_{\infty}\right) \cap M, \forall r, \varepsilon>0, \exists h_{0} \in \mathbf{N}: \forall h \geq h_{0}$,

$$
\inf \left\{\widehat{f}_{h}(v): v \in B(u, r) \cap M\right\}<\widehat{f}_{\infty}(u)+\varepsilon .
$$

Let $r, \varepsilon>0$ and let $v \in \partial^{-} I_{M}(u)$ with $|v|=1$. By iv), vi),

Remarks 2.9 and 2.11. - there exist $u^{+}, u^{-} \in D\left(\widehat{f}_{\infty}\right)$ such that $\left.v \mid u^{+}-u\right)>0,\left(v \mid u^{-}-u\right)<0$. By substituting $u^{ \pm}$with $\left(s u^{ \pm}+(1-s) u\right)$ for some small $s$ in $] 0,1]$, we can also assume that $q\left|u^{+}-u^{-}\right|^{2}<2 \varepsilon$, $\hat{f}_{\infty}\left(u^{ \pm}\right)<\widehat{f}_{\infty}(u)+\varepsilon / 2$ and that for every $v^{ \pm}$in a neighborhood of $u^{ \pm}$ $\left(t v^{+}+(1-t) v^{-} \in M \cap B(u, r)\right.$ for some $t \in[0,1]$.

Let $\left(u_{h}^{+}\right)$and $\left(u_{h}^{-}\right)$be two sequences in $H$ such that $\lim _{h} u_{h}^{ \pm}=u^{ \pm}$, $\lim _{h} \hat{f}_{h}\left(u_{h}^{ \pm}\right)=\widehat{f}_{\infty}\left(u^{ \pm}\right)$. Then

$\left(t_{h} u_{h}^{+}+\left(1-t_{h}\right) u_{h}^{-}\right) \in M \cap B(u, r)$ for some $t_{h} \in[0,1]$

eventually as $h \rightarrow+\infty$ and by iv) we have

$$
\begin{gathered}
\widehat{f}_{h}\left(t_{h} u_{h}^{+}+\left(1-t_{h}\right) u_{h}^{-}\right) \leq \\
\leq t_{h} \widehat{f}_{h}\left(u_{h}^{+}\right)+\left(1-t_{h}\right) \widehat{f}_{h}\left(u_{h}^{-}\right)+t_{h}\left(1-t_{h}\right) q\left|u_{h}^{+}-u_{h}^{-}\right|^{2}< \\
<t_{h} \widehat{f}_{h}\left(u_{h}^{+}\right)+\left(1-t_{h}\right) \widehat{f}_{h}\left(u_{h}^{-}\right)+\varepsilon / 2<\widehat{f}_{\infty}(u)+\varepsilon .
\end{gathered}
$$

Finally, let us prove ix). By paracompactness and partition of unity, it is sufficient to show that for every $(\bar{u}, \bar{x}) \in\left(\bigcup_{h \in N} D\left(\widetilde{f}_{h}\right)\right) \times \mathbf{R}$ there exist $\varepsilon>0$, $K \in \mathbf{R}$ such that

$$
\tilde{f}_{h}(v) \geq \tilde{f}_{h}(u)+(\alpha \mid v-u)-K(1+|\alpha|)|v-u|^{2}
$$

whenever $h \in \mathbf{N}, v \in H, u \in D\left(\partial^{-} \tilde{f}_{h}\right), \alpha \in \partial^{-} \tilde{f}_{h}(u),|u-\leq u|<\varepsilon$, $\left|\tilde{f}_{h}(u)-\leq x\right|<\varepsilon$. 


\section{Degiovanni}

By contradiction, we could find $(\bar{u}, \bar{x})$ and sequences $\left.\left(h_{k}\right),\left(u_{k}\right),\left(v_{k}\right), \alpha_{k}\right)$ such that

$$
\tilde{f}_{h_{k}}\left(v_{k}\right)<\tilde{f}_{h_{k}}\left(u_{k}\right)+\left(\alpha_{k} \mid v_{k}-u_{k}\right)-k\left(1+\left|\alpha_{k}\right|\right)\left|v_{k}-u_{k}\right|^{2}
$$

$\alpha_{k} \in \partial^{-} \tilde{f}_{h_{k}}\left(u_{k}\right),\left|u_{k}-\bar{u}\right|<1 / k,\left|\tilde{f}_{h_{k}}\left(u_{k}\right)-\bar{x}\right|<1 / k$.

$\mathrm{Up}_{\mathrm{p}}$ to a subsequence, we have that $\lim _{k} h_{k}=h_{\infty} \in \overline{\mathbf{N}}$. By lower semicontinuity and $\Gamma$-convergence, we deduce that $\bar{u} \in D\left(\tilde{f}_{h_{\infty}}\right) \cap M$. By iii), vi) and (2.18) we can apply Theorem 2.12 obtaining $\alpha_{k}=\beta_{k}+\eta_{k}$ with $\beta_{k} \in \partial^{-} \widehat{f}_{h_{k}}\left(u_{k}\right), \eta_{k} \in \partial^{-} I_{M}\left(u_{k}\right)$.

We claim that

$$
\limsup _{k} \frac{\left|\eta_{k}\right|}{1+\left|\alpha_{k}\right|}<+\infty .
$$

Of course we can assume that $\eta_{k} \neq 0$. By Remark 2.9, up to a subsequence $\left(\eta_{k} /\left|\eta_{k}\right|\right)$ converges to some $\nu \in \partial^{-} I_{M}(\bar{u})$ with $|\nu|=1$. By vi) and Remark 2.11 there exists $w \in D\left(\widehat{f}_{h_{\infty}}\right)$ such that $(\nu \mid w-\bar{u})<0$. By $\Gamma$-convergence, there exists $\left(w_{k}\right)$ converging to $w$ with $\lim _{k} \widehat{f}_{h_{k}}\left(w_{k}\right)=\widehat{f}_{h_{\infty}}(w)$. By $(2.18)$ we conclude that

$$
\widehat{f}_{h_{k}}\left(w_{k}\right) \geq \widehat{f}_{h_{k}}\left(u_{k}\right)+\left(\alpha_{k}-\eta_{k} \mid w_{k}-u_{k}\right)-q\left|w_{k}-u_{k}\right|^{2}
$$

namely

$$
\begin{gathered}
\left|\eta_{k}\right|\left(\frac{\eta_{k}}{\left|\nu_{k}\right|} \mid w_{k}-u_{k}\right) \geq \\
\geq-\widehat{f}_{h_{k}}\left(w_{k}\right)+\widehat{f}_{h_{k}}\left(u_{k}\right)+\left(\alpha_{k} \mid w_{k}-u_{k}\right)-q\left|w_{k}-u_{k}\right|^{2}
\end{gathered}
$$

which gives the result.

Now we remark that $v_{k} \neq u_{k}$ and

$$
\limsup \frac{\left|\left(\left(\eta_{k} /\left|\eta_{k}\right|\right) \mid v_{k}-u_{k}\right)\right|}{\left|v_{k}-u_{k}\right|^{2}}<+\infty,
$$

because $M$ is of class $C_{\text {loc }}^{1,1}$.

Then, taking into account (2.18), we conclude that

$$
\begin{gathered}
K<\frac{-\widehat{f}_{h_{k}}\left(v_{k}\right)+\widehat{f}_{h_{k}}\left(u_{k}\right)+\left(\beta_{k}+\eta_{k} \mid v_{k}-u_{k}\right)}{\left(1+\left|\alpha_{k}\right|\right)\left|v_{k}-u_{k}\right|^{2}} \leq \\
\leq \frac{q\left|v_{k}-u_{k}\right|^{2}+\left|\left(\eta_{k} \mid v_{k}-u_{k}\right)\right|}{\left(1+\left|\alpha_{k}\right|\right)\left|v_{k}-u_{k}\right|^{2}}= \\
=\frac{q}{1+\left|\alpha_{k}\right|}+\frac{\left|\eta_{k}\right|}{1+\left|\alpha_{k}\right|} \frac{\left|\left(\left(\eta_{h} /\left|\eta_{k}\right|\right) \mid v_{k}-u_{k}\right)\right|}{\left|v_{k}-u_{k}\right|^{2}} \\
-226-
\end{gathered}
$$


Bifurcation problems for nonlinear elliptic variational inequalities

which is absurd.

We point out that a property like ix) was already proved in $[8$, Theorem 1.13] for a single function $f$.

\section{Variational bifurcation for nonsmooth functions}

Throughout this section we keep the notations of $\S 2$. We shall consider a real Hilbert space $H$, a convex open subset $W$ of $H$ with $0 \in W$, a function

$$
f: \bar{W} \rightarrow \mathbf{R} \cup\{+\infty\}
$$

such that

$$
f(0)=0, \quad 0 \in \partial^{-} f(0)
$$

and a symmetric bounded linear operator

$$
L: H \rightarrow H \text {. }
$$

Our purpose is to study the set of the pairs $(\lambda, u)$ such that

$$
\left\{\begin{array}{l}
(\lambda, u) \in \mathbf{R} \times W \\
\lambda L u \in \partial^{-} f(u)
\end{array}\right.
$$

Because of (3.1), for every $\lambda$ in $\mathbf{R}$ the pair $(\lambda, 0)$ satisfies (3.2).

Definition 3.3. - A real number $\lambda$ is said to be of bifurcation for (3.2), if there exists a sequence $\left(\left(\lambda_{h}, u_{h}\right)\right)$ or solutions of (3.2) with $u_{h} \neq 0$ and

$$
\lim _{h}\left(\lambda_{h}, u_{h}\right)=(\lambda, 0)
$$

As in the case of smooth functions $f$ (see [22]), we want to compare the bifurcation values with the eigenvalues of some "linearized" problem. In order to carry out such a program, we make the following further assumptions on $f$ :

(A1) the function $f$ is lower semicontinuous on $\bar{W}$ and there exists $q \in \mathrm{R}^{+}$such that the function $\left\{u \longmapsto f(u)+q|u|^{2}\right\}$ is convex on $\bar{W}$;

(A2) there exists a function $f_{0}: H \rightarrow \mathbf{R} \cup\{+\infty\}$ such that for every sequence $\left(\rho_{h}\right)$ in $\left.] 0,1\right]$ converging to zero, we have

$$
\begin{gathered}
f_{0}=\Gamma^{-}(H) \lim _{h} f_{\rho_{h}} \\
-227-
\end{gathered}
$$




\section{Degiovanni}

where $\forall \rho \in] 0,1], f_{\rho}: H \rightarrow \mathbf{R} \cup\{+\infty\}$ is defined by

$$
f_{\rho}(u)=\left\{\begin{array}{ll}
\rho^{-2} f(\rho u) & \rho u \in \bar{W} \\
+\infty & \rho u \in H \backslash \bar{W}
\end{array} .\right.
$$

Proposition 3.4. - Let $\rho \in] 0,1]$. Then the following facts

hold :

i) $f_{\rho}$ is lower semicontinuous on $H$ and the function $\left\{u \longmapsto f_{\rho}(u)+q|u|^{2}\right\}$ is convex on $H$;

ii) $\forall v \in H, \forall u \in D\left(\partial^{-} f_{\rho}\right), \forall \alpha \in \partial^{-} f_{\rho}(u)$, we have

$$
f_{\rho}(v) \geq f_{\rho}(u)+(\alpha \mid v-u)-q|v-u|^{2}
$$

iii) $f_{\rho}(0)=0,0 \in \partial^{-} f_{\rho}(0)$;

iv) $\forall u, \alpha \in H$, we have

$u \in D\left(\partial^{-} f_{\rho}\right)$ and $\alpha \in \partial^{-} f_{\rho}(u) \Leftrightarrow \rho u \in D\left(\partial^{-} f\right)$ and $\rho \alpha \in \partial^{-} f(\rho u)$;

$v)$ for every sequence. $\left(\rho_{h}\right)$ in $\left.] 0,1\right]$ converging to $\rho$, we have

$$
f_{\rho}=\Gamma^{-}(H) \lim _{h} f_{\rho_{h}} .
$$

Proof.-Properties i), iii), iv) and v) are immediate consequences of the definition of $f_{\rho}$. Property ii) is an easy consequence of propery i).

Proposition 3.5. - Let $\left(\rho_{h}\right)$ be a sequence in [0,1], $\left(u_{h}\right)$ and $\left(\alpha_{h}\right)$ two sequences in $H$. Let us suppose that $\left(\rho_{h}\right)$ converges to $\rho \in[0,1]$, $\left(u_{h}\right)$ converges to $u \in H,\left(\alpha_{h}\right)$ converges weakly to $\alpha \in H$ and that $\alpha_{h} \in \partial^{-} f_{\rho_{h}}\left(u_{h}\right), \forall h \in \mathbf{N}$. Then we have

$$
\alpha \in \partial^{-} f_{\rho}(u), \quad f_{\rho}(u)=\lim _{h} f_{\rho_{h}}\left(u_{h}\right) .
$$

Proposition 3.6. - The following facts hold:

i) $f_{0}$ is lower semicontinuous on $H$ and the function

$$
\left\{u \longmapsto f_{0}(u)+q|u|^{2}\right\} \text { is convex on } H \text {; }
$$

ii) $\forall v \in H, \forall u \in D\left(\partial^{-} f_{0}\right), \forall \alpha \in \partial^{-} f_{0}(u)$, we have

$$
\begin{aligned}
f_{0}(v) \geq f_{0}(u)+ & (\alpha \mid v-u)-q|v-u|^{2} \\
& -228-
\end{aligned}
$$


Bifurcation problems for nonlinear elliptic variational inequalities

iii) $f_{0}(0)=0,0 \in \partial^{-} f_{0}(0)$;

iv) $\forall s>0, \forall u \in H, f_{0}(s u)=s^{2} f_{0}(u)$;

v) $\forall s>0, \forall u \in D\left(\partial^{-} f_{0}\right), \forall \alpha \in \partial^{-} f_{0}(u), s \alpha \in \partial^{-}(s u)$;

vi) $\forall u \in D\left(\partial^{-} f_{0}\right), \forall \alpha \in \partial^{-} f_{0}(u),(\alpha \mid u)=2 f_{0}(u)$.

Proof 9.5 and 3.6. - Property (3.6) $)_{\mathrm{i}}$ is a simple consequence of Proposition $(3.4)_{\mathrm{i}}$ and the definition of $\Gamma$-convergence; property $(3.6)_{\mathrm{ii}}$ is an easy consequence of $(3.6)_{i}$.

Let us prove Proposition 3.5. By Proposition $3.4_{\mathrm{ii}}$ and (3.6) $)_{\mathrm{ii}}$ we have

$$
\forall v \in H, f_{\rho_{h}}(v) \geq f_{\rho_{h}}\left(u_{h}\right)+\left(\alpha_{h} \mid v-u_{h}\right)-q\left|v-u_{h}\right|^{2} .
$$

By (A2) and Proposition 3.4v we can choose $v=z_{h}$, where $\lim _{h} z_{h}=u$ and $\lim _{h} f_{\rho_{h}}\left(z_{h}\right)=f_{\rho}(u)$, obtaining

$$
f_{\rho}(u) \geq \underset{h}{\limsup } f_{\rho_{h}}\left(u_{h}\right)
$$

By the definition of $\Gamma$-convergence we conclude that

$$
f_{\rho}(u)=\lim _{h} f_{\rho_{h}}\left(u_{h}\right) .
$$

Now $\forall w \in H$ we choose $v=w_{h^{\prime}}$ where $\lim _{h} w_{h}=w, \lim _{h} f_{\rho_{h}}\left(w_{h}\right)=f_{\rho}(w)$, obtaining

$$
\forall w \in H, f_{\rho}(w) \geq f_{\rho}(u)+(\alpha \mid w-u)-q|w-u|^{2}
$$

which implies $\alpha \in \partial^{-} f_{\rho}(u)$.

Property $(3.6)_{\mathrm{iii}}$ is a consequence of Propositions $(3.4)_{\mathrm{iii}}$ and 3.5.

Let us prove $(3.6)_{\mathrm{iv}}$. Let $\left(\rho_{h}\right)$ be a sequence in $\left.] 0,1\right]$ converging to zero and let $\left(u_{h}\right)$ be a sequence such that $\lim _{h} u_{h}=u, \lim _{h} f_{\rho_{h}}\left(u_{h}\right)=f_{0}(u)$.

Since

$$
\begin{gathered}
f_{s^{-1} \rho_{h}}\left(s u_{h}\right)=s^{2} f_{\rho_{h}}\left(u_{h}\right), \\
\lim _{h} s^{-1} \rho_{h}=0, \quad \lim _{h} s u_{h}=s u,
\end{gathered}
$$

by (A2) we have

$$
f_{0}(s u) \leq \liminf _{h} f_{s^{-1} \rho_{h}}\left(s u_{h}\right)=s^{2} f_{0}(u)
$$


For the same reason

$$
f_{0}(u)=f_{0}\left(s^{-1}(s u)\right) \leq s^{-2} f_{0}(s u)
$$

Therefore $(3.6)_{\text {iv }}$ is proved.

Property $(3.6)_{\mathrm{v}}$ is an easy consequence of $(3.6)_{\mathrm{iv}}$.

Finally, let us prove $(3.6)_{\mathrm{vi}}$. By $(3.6)_{\mathrm{vi}}$ we have

$$
\begin{gathered}
(\alpha \mid u) \leq \liminf _{t \rightarrow 0^{+}} \frac{f_{0}(u+t u)-f_{0}(u)}{t}= \\
=\liminf _{t \rightarrow 0^{+}}(2+t) f_{0}(u)=2 f_{0}(u) .
\end{gathered}
$$

On the other hand

$$
-(\alpha \mid u) \leq \liminf _{t \rightarrow 0^{+}} \frac{f_{0}(u-t u)-f_{0}(u)}{t}=-2 f_{0}(u) .
$$

The function $f_{0}(u)$ introduced in (A2) plays the role of the quadratic form $\frac{1}{2} f^{\prime \prime}(0)(u, u)$ in the smooth case. In the following it will be convenient to consider also the "linearized" problem

$$
\left\{\begin{array}{l}
(\lambda, u) \in \mathbf{R} \times H \\
\lambda L u \in \partial^{-} f_{0}(u)
\end{array}\right.
$$

Remark 3.8. - For every $\lambda$ in $\mathbf{R}$ the set.

$$
\left\{u \in H: \lambda L u \in \partial^{-} f_{0}(u)\right\}
$$

is a closed cone.

Proof. - It is a consequence of Propositions 3.5 and (3.6) $\mathrm{v}$.

It is not true, in general, that $\left\{u \in H: \lambda L u \in \partial^{-} f_{0}(u)\right\}$ is convex. Take, for instance, $H=W=\mathbf{R}^{2}, f(x, y)=\left(x^{4}+y^{4}\right)^{1 / 2}$ and $L$ the identity map. Then (3.1), (A1) and (A2) are satisfied with $f_{0}(x, y)=\left(x^{4}+y^{4}\right)^{1 / 2}$, but for $\lambda=\sqrt{2}$ we have

$$
\left\{u \in H: \lambda L u \in \partial^{-} f_{0}(u)\right\}=\left\{(x, y) \in \mathbf{R}^{2}: x^{2}=y^{2}\right\}
$$

and for $\lambda=2$ we have

$$
\left\{u \in H: \lambda L u \in \partial^{-} f_{0}(u)\right\}=\left\{(x, y) \in \mathbf{R}^{2}: x y=0\right\}
$$


Bifurcation problems for nonlinear elliptic variational inequalities

DEFINITION 3.9. - A real number $\lambda$ is said to be an eigenvalue of (3.7), if the pair $\lambda, u$ ) satisfies (3.7) for some $u \neq 0$.

Remark 3.10. - If $(\lambda, u)$ satisfies (3.7), then $f_{0}(u)=\frac{1}{2} \lambda(L u \mid u)$.

Proof.- - It is a consequence of Proposition (3.6) $)_{\mathrm{vi}}$.

Now it is convenient to introduce the sets

$$
\begin{gathered}
M^{+}=\left\{u \in H: \frac{1}{2}(L u \mid u)=1\right\} \\
M^{-}=\left\{u \in H: \frac{1}{2}(L u \mid u)=-1\right\},
\end{gathered}
$$

which are hypersurfaces in $H$ of class $C^{\infty}$ and closed subsets of $H$.

According to Remark 2.9, we have for every $u$ in $M^{ \pm}$,

$$
\partial^{-} I_{M^{ \pm}}(u)=\{\lambda L u: \lambda \in \mathbf{R}\}
$$

Proposition 3.11. - For every $u \in D\left(f_{0}\right) \cap M^{ \pm}, D\left(f_{0}\right)$ and $M^{ \pm}$are not tangent at $u$.

Proof.- Let $u \in D\left(f_{0}\right) \cap M^{+}$. The set $D\left(f_{0}\right)$ is a convex cone by Proposition (3.6) $)_{\mathrm{i}}$ and $(3.6)_{\mathrm{iv}}$. If we take $u^{+}=2 u, u^{-}=0$, we have

$$
\left(L u \mid u^{+}-u\right)=2, \quad\left(L u \mid u^{-}-u\right)=-2 .
$$

By remark $2.11 D\left(f_{0}\right)$ and $M^{+}$are not tangent at $u$. The same argument works for $M^{-}$.

Proposition 3.12.- Given a $\lambda \in \mathbf{R}$, let us consider the following facts :

i) $(\lambda, u)$ satisfies (3.7) for some $u$ with $(L u \mid u)>0$;

ii) $\lambda$ is critical from below for $\left(f_{0}+I_{M^{+}}\right)$;

iii) $(\lambda, u)$ satisfies (3.7) for some $u$ with $(L u \mid u)<0$;

iv) $-\lambda$ is critical from below for $\left(f_{0}+I_{M^{-}}\right)$.

Then we have

$$
\begin{aligned}
& \text { i) } \Leftrightarrow \text { ii) ; } \\
& \text { iii) } \Leftrightarrow \text { iv). }
\end{aligned}
$$


Proof.-By Propositions $(3.6)_{\mathrm{i}},(3.6)_{\mathrm{ii}}$ and 3.11 we can apply Theorem 2.12 , obtaining for every $v$ in $D\left(f_{0}\right) \cap M^{+}$,

$$
\partial^{-}\left(f_{0}+I_{M^{+}}\right)(v)=\left\{\alpha-\lambda L v: \alpha \in \partial^{-} f_{0}(v), \lambda \in \mathbf{R}\right\}
$$

Now let us prove that i) $\Rightarrow$ ii). Let $(\lambda, u)$ be a solution of (3.7) with $(L u \mid u)>0$ and let

$$
v=\left(\frac{1}{2}(L u \mid u)\right)^{-1 / 2} u .
$$

By Remark 3.8 $\lambda L v \in \partial^{-} f_{0}(v)$. Moreover $v \in M^{+}$and $\left(f_{0}+I_{M^{+}}\right)(v)=\lambda$ by Remark 3.10. By (3.13) we conclude that $0 \in \partial^{-}\left(f_{0}+I_{M^{+}}\right)(v)$.

Let us prove that ii) $\Rightarrow$ i). Let $v \in M^{+}$be such that $f_{0}(v)=\lambda$, $0 \in \partial^{-}\left(f_{0}+I_{M^{+}}\right)(v)$. Of course $(L v \mid v)>0$. By (3.13) there exists $\mu \in \mathbf{R}$ such that $\mu L v \in \partial^{-} f_{0}(v)$. By Remark 3.10 we conclude that $\lambda=f_{0}(v)=\mu$.

The proof that iii) $\leftrightarrow$ iv) is analogous.

THEOREM 3.14. - Let us assume that for every sequence $\left(u_{h}\right)$ in $W \backslash\{0\}$ with

$$
\lim _{h}=u_{h}=0, \quad \sup _{h}\left|u_{h}\right|^{-2} f\left(u_{h}\right)<+\infty,
$$

the sequence $\left(u_{h} /\left|u_{h}\right|\right)$ has a convergent subsequence.

Then every $\lambda$ of bifurcation for (3.2) is an eigenvalue of (3.7).

Proof. - Let $\left(\left(\lambda_{h}, u_{h}\right)\right)$ be a sequence as in Definition 3.3 and let $\rho_{h}=$ $\left|u_{h}\right|, v_{h}=u_{h} /\left|u_{h}\right|$. By proposition (3.4) $)_{\mathrm{iv}}$.

$$
\lambda_{h} L v_{h} \in \partial^{-} f_{\rho_{h}}\left(v_{h}\right) .
$$

Moreover by Proposition (3.4) $\mathrm{ii}$ and (3.4) $)_{\mathrm{iii}}$

$$
0=f_{\rho_{h}}(0) \geq f_{\rho_{h}}\left(v_{h}\right)-\lambda_{h}\left(\cdot L v_{h} \mid v_{h}\right)-q\left|v_{h}\right|^{2} .
$$

Since $\left|v_{h}\right|=1$, we have

$$
\sup _{h}\left|u_{h}\right|^{-2} f\left(u_{h}\right)=\sup _{h} f_{\rho_{h}}\left(v_{h}\right)<+\infty .
$$

Therefore, up to a subsequence, $\left(v_{h}\right)$ converges to some $v$ in $H$ with $|v|=1$.

By Proposition 3.5 we conclude that

$$
\lambda L v \in \partial^{-} f_{0}(v) .
$$


Bifurcation problems for nonlinear elliptic variational inequalities

The converse is not true, in general. Let us take the following counterexample from [30].

Let $H=\mathbf{R}^{2}, W=B(0,1), f: \bar{W} \rightarrow \mathbf{R} \cup\{+\infty\}$ defined by

$$
f(x, y)= \begin{cases}\frac{1}{2} x^{2}+y^{2}-x y^{2} & x \geq 0 \\ +\infty & \text { elsewhere }\end{cases}
$$

and $L$ the identity map. Then all the assumptions of Theorem 3.14 are satisfied with

$$
f_{0}(x, y)= \begin{cases}\frac{1}{2} x^{2}+y^{2} & x \geq 0 \\ +\infty & \text { elsewhere }\end{cases}
$$

On the other hand $\lambda=2$ is an isolated eigenvalue of (3.7) which is not of bifurcation for (3.2).

From our point of view, the feature of this example is that $\lambda$, which is critical from below for $\left(f_{0}+I_{M^{+}}\right)$by Proposition 3.12, is not essential for $\left(f_{0}+I_{M^{+}}\right)$in the sense of Definition 2.8 .

Roughly speaking, the first purpose of this section is to show that, if $\lambda \in \mathbf{R}$ is an eigenvalue of (3.7) and $\lambda$ is essential for $\left(f_{0}+I_{M^{+}}\right)$or $-\lambda$ is essential for $\left(f_{0}+I_{M^{-}}\right)$, then $\lambda$ is of bifurcation for (3.2). On the contrary, we shall not treat the eigenvalues $\lambda$ such that

$$
\forall u \in H, \lambda L u \in \partial^{-} f_{0}(u) \Rightarrow(L u \mid u)=0 .
$$

First of all let us consider the following compactness assumptions :

$\left(\mathrm{A}^{+}\right)$for every sequence $\left(u_{h}\right)$ in $\bar{W}$ with $\left.\left.\frac{1}{2}\left(L u_{h} \mid u_{h}\right) \in\right] 0,1\right]$ and

$$
\sup _{h}\left|\left(L u_{h} \mid u_{h}\right)\right|^{-1} f\left(u_{h}\right)<+\infty
$$

the sequence $\left.\left.\left(\left|L u_{h}\right| u_{h}\right)\right|^{-1 / 2} u_{h}\right)$ has a convergent subsequence;

$\left(\mathrm{A}^{-}\right)$for every sequence $\left(u_{h}\right)$ in $\bar{W}$ with $\frac{1}{2}\left(L u_{h} \mid u_{h}\right) \in[-1,0[$ and

$$
\sup _{h}\left|\left(L u_{h} \mid u_{h}\right)\right|^{-1} f\left(u_{h}\right)<+\infty
$$

the sequence $\left(\left|\left(L u_{h} \mid u_{h}\right)\right|^{-1 / 2} u_{h}\right)$ has a convergent subsequence.

If $L$ is the identity map of $H$, hypothesis $\left(\mathrm{A}^{+}\right)$implies the compactness assumption made in Theorem 3.14. 
Proposition 3.15. - Condition $\left(A 3^{+}\right)$(resp. $\left.\left(A 3^{-}\right)\right)$holds if and only if for every sequence $\left(\rho_{h}\right)$ in $\left.] 0,1\right]$ the sequence $\left(f_{\rho_{h}}+I_{M+}\right)\left(\operatorname{resp} .\left(f_{\rho_{h}}+I_{M^{-}}\right)\right)$ is equicoercive.

Proof. - Let $\left(\mathrm{A} 3^{ \pm}\right)$hold. Let $c \in \mathbf{R}$ and let $\left(u_{k}\right)$ be a sequence in $M^{ \pm}$ with $f_{\rho_{h_{k}}}\left(u_{k}\right) \leq c$ for some sequence $\left(h_{k}\right)$ in $\mathbf{N}$.

If we set $v_{k}=\rho_{h_{k}} u_{k}$, we have

$$
\frac{1}{2}\left(L v_{k} \mid v_{k}\right)= \pm \rho_{h_{k}}^{2}, \quad\left|\left(L v_{k} \mid v_{k}\right)\right|^{-1} f\left(v_{k}\right)=\frac{1}{2} f_{\rho_{h_{k}}}\left(u_{k}\right) \leq c / 2 .
$$

$\mathrm{By}\left(\mathrm{A} 3^{ \pm}\right),\left(u_{k}\right)$ has a convergent subsequence.

Now let us assume that for every sequence $\left(\rho_{h}\right)$ in $\left.] 0,1\right]$ the sequence $\left(f_{\rho_{h}}+I_{M^{ \pm}}\right)$is equicoercive.

Let $\left(u_{h}\right)$ be a sequence as in $\left(\mathrm{A}^{ \pm}\right)$. Let $\rho_{h}=\left(\frac{1}{2}\left|\left(L u_{h} \mid u_{h}\right)\right|\right)^{1 / 2}$ and $v_{h}=u_{h} / \rho_{h}$. Then $v_{h} \in M^{ \pm}$and

$$
\sup _{h} f_{\rho_{h}}\left(v_{h}\right)=2 \sup _{h}\left|\left(L u_{h} \mid u_{h}\right)\right|^{-1} f\left(u_{h}\right)<+\infty,
$$

Therefore $\left(v_{h}\right)$ has a convergent subsequence.

Proposition 3.16. - Let $\left(A 3^{+}\right)$(resp. $\left(A 3^{-}\right)$) hold. Then for every $c$ in $\mathbf{R}$ the set $\left(f_{0}+I_{M^{+}}\right)^{c}$ (resp. $\left.\left(f_{0}+I_{M^{-}}\right)^{c}\right)$ is compact.

Proof. - Let $\left(\rho_{h}\right)$ be a sequence in 10,1$]$ converging to zero and let $\rho_{\infty}=0$. By Propositions $(3.4)_{i},(3.6)_{i},(\mathrm{~A} 2),\left(\mathrm{A} 3^{ \pm}\right)$and Proposition 3.11, we can apply Theorem 2.17 to the sequence $\left(f_{\rho_{h}}\right)$ and the hypersurface $M^{ \pm}$. Then $\left(f_{0}+I_{M^{ \pm}}\right)$is the $\Gamma$-limit of some equicoercive sequence of functions by Theorem $(2.17)_{\mathrm{i}},(2.17)_{\mathrm{x}}$ and $(2.17)_{\mathrm{xi}}$. By Remark 2.15 the thesis follows.

From now on we restrict our attention to the eigenvalues $\lambda$ such that $\lambda L u \in \partial^{-} f_{0}(u)$ for some $u$ with $(L u \mid u) \neq 0$. For sake of simplicity, we shall consider only eigenvalues $\lambda$ such that $\lambda L u \in \partial^{-} f(u)$ for some $u$ with $(L u \mid u)>0$. By changing $L$ in $-L$ and $\lambda$ in $-\lambda$, we can always reduce ourselves to this case.

Proposition 3.17. - Let $\left(\mathrm{AS}^{+}\right)$hold and let $\lambda \in \mathbf{R}$. Let us assume that $E:=\left\{u \in H: \lambda L u \in h^{-} f_{0}(u)\right\}$ is convex and that $\left(L u_{0} \mid u_{0}\right)>0$ for some $u_{0} \in E$. Then the set $\{u \in E:|u|=1\}$ is compact and we have

$$
\min \{(L u \mid u): u \in E,|u|=1\}>0 .
$$


Bifurcation problems for nonlinear elliptic variational inequalities

Proof.- By proposition (3.6) $)_{\mathrm{vi}}, \forall u \in E \cap M^{+}, f_{0}(u)=\lambda$. By Proposition $3.16 E \cap M^{+}$is compact, hence bounded.

We claim that $\forall u \in E \backslash\{0\},(L u \mid u)>0$. By contradiction, let $v \in E \backslash\{0\}$ with $(L v \mid v) \leq 0$. Since $u_{0}$ and $v$ are linearly independent, we have

$$
\lim _{h}\left(\min _{0 \leq t \leq 1}\left|(1-t) h u_{0}+t h v\right|\right)=+\infty
$$

Since $E$ is a cone by Remark 3.8, eventually as $h \rightarrow \infty$ we can find $w_{h} \in E \cap M^{+}$of the form $w_{h}=\left(1-t_{h}\right) h u_{0}+t_{h} h v$ for some $t_{h} \in[0,1]$. Then $\lim _{h}\left|w_{h}\right|=+\infty$, which is a contradiction.

To conclude the proof, it is sufficient to remark that the map

$$
\begin{aligned}
E \cap M^{+} & \rightarrow\{u \in E:|u|=1\} \\
u & \longmapsto u /|u|
\end{aligned}
$$

is a homeomorphism.

COROLlARY 3.18. - Let either $\left(A 3^{+}\right)$or $\left(A 3^{-}\right)$hold and let us assume that for every $\lambda$ in $\mathbf{R}$ the set $\left\{u \in H: \lambda L u \in \partial^{-} f_{0}(u)\right\}$ is convex. Then the sets

$\{\lambda \in \mathbf{R}:(\lambda, u))$ satisfies (3.7) for some $u$ with $(L u \mid u)>0\}$

and

$\{\lambda \in \mathbf{R}:(\lambda, u)$ satisfies (3.7) for some $u$ with $(L u \mid u)<0\}$

are disjoint.

Proof.- By changing $L$ in $-L$, we can suppose that $\left(\mathrm{A}^{+}\right)$holds. Then the thesis follows from Proposition 3.17.

Lemma 3.19. - Let $\left(\mathrm{A}^{+}\right)$hold and let $\lambda \in \mathbf{R}$. Then the following facts are equivalent :

i) there exists $\rho_{0}>0, \quad\left\{\lambda_{\rho}: 0<\rho \leq \rho_{0}\right\} \subset \mathbf{R}$, $\left\{u_{\rho}: 0<\rho \leq \rho_{0}\right\} \subset W$ such that

$$
\begin{gathered}
\left.\forall \rho \in] 0, \rho_{0}\right], \lambda_{\rho} L u_{\rho} \in \partial^{-} f\left(u_{\rho}\right) \text { and } \frac{1}{2}\left(L u_{\rho} \mid u_{\rho}\right)=\rho^{2} ; \\
\lim _{\rho 0^{+}}\left(\lambda_{\rho}, u_{\rho}\right)=(\lambda, 0), \lim _{\rho \rightarrow O^{+}} f\left(u_{\rho}\right) / \rho^{2}=\lambda ;
\end{gathered}
$$


ii) for every sequence $\left(\rho_{h}\right)$ in $\left.] 0,1\right]$ converging to zero, there exists $h_{0} \in$ $\mathbf{N}, \quad\left\{\lambda_{h}: h \geq h_{0}\right\} \subset \mathbf{R}, \quad\left\{u_{h}: h \geq h_{0}\right\} \subset M^{+}$such that

$$
\begin{gathered}
\forall h \geq h_{0}, \lambda_{h} L u_{h} \in \partial^{-} f_{\rho_{h}}\left(u_{h}\right) ; \\
\lim _{h} f_{\rho_{h}}\left(u_{h}\right)=\lambda ;
\end{gathered}
$$

iii) for every $\varepsilon>0$ and for every sequence $\left(\rho_{h}\right)$ in $\left.] 0,1\right]$ converging to zero, there exists $h_{0} \in \mathbf{N},\left\{\lambda_{h}: h \geq h_{0}\right\} \subset \mathbf{R},\left\{u_{h}: h \geq h_{0}\right\} \subset M^{+}$such that

$$
\forall h \geq h_{0}, \lambda_{h} L u_{h} \in \partial^{-} f_{\rho_{h}}\left(u_{h}\right) \text { and } \lambda-\varepsilon \leq f_{\rho_{h}}\left(u_{h}\right) \leq \lambda+\varepsilon .
$$

Proof. - Let us prove that ii) $\Rightarrow$ i). First of all let $\left(\rho_{h}\right),\left(\lambda_{h}\right)$ and $\left(u_{h}\right)$ be as in ii). We claim that $\lim _{h} \lambda_{h}=\lambda$.

In fact by Proposition 3.15, up to a subsequence $\left(u_{h}\right)$ converges to some $u$ in $M^{+}$. By (A2) we conclude that $u \in D\left(f_{0}\right)$. Again by (A2) and Proposition $(3.6)_{\text {iv }}$, for every $s>0$ we can find a sequence $\left(w_{h}\right)$ converging to $s u$ with $\lim _{h} f_{\rho_{h}}\left(w_{h}\right)=f_{0}(s u)=s^{2} f_{0}(u)$. By proposition $(3.4)_{i i}$ we have

$$
\lambda_{h}\left(L u_{h} \mid w_{h}-u_{h}\right) \leq f_{\rho_{h}}\left(w_{h}\right)-f_{\rho_{h}}\left(u_{h}\right)+q\left|w_{h}-u_{h}\right|^{2} .
$$

On the other hand

$$
\begin{gathered}
\lim _{h}\left(L u_{h} \mid w_{h}-u_{h}\right)=(s-1)(L u \mid u)=2(s-1), \\
\lim _{h}\left(f_{\rho_{h}}\left(w_{h}\right)-f_{\rho_{h}}\left(u_{h}\right)\right)=s^{2} f_{0}(u)-\lambda, \\
\lim _{h}\left|w_{h}-u_{h}\right|^{2}=(s-1)^{2}|u|^{2} .
\end{gathered}
$$

Since we can choose $s$ in $] 0,1[$ and in $] 1,+\infty[$, we conclude first of all that

$$
\underset{h}{\limsup }\left|\lambda_{h}\right|<+\infty \text {. }
$$

By Proposition 3.5 we deduce that $f_{0}(u)=\lambda$. Therefore, because of the arbitrary of $s$,

$$
\lim _{h} \lambda_{h}=\lambda \text {. }
$$

Moreover, if we set $v_{h}=\rho_{h} u_{h}$, we have, taking also into account Proposition $(3.4)_{\mathrm{iv}}$,

$$
\begin{gathered}
\forall h \geq h_{0}, \lambda_{h} L v_{h} \in \partial^{-} f\left(v_{h}\right) \text { and } \frac{1}{2}\left(L v_{h} \mid v_{h}\right)=\rho_{h}^{2}, \\
\lim _{h}\left(\lambda_{h}, v_{h}\right)=(\lambda, 0), \lim _{h} f\left(v_{h}\right) / \rho_{h}^{2}=\lambda . \\
-236-
\end{gathered}
$$


Bifurcation problems for nonlinear elliptic variational inequalities

Now, arguing by contradiction, it is easy to see that ii) $\Rightarrow$ i).

The proof that iii) $\Rightarrow$ ii) is trivial. Let us prove that i) $\Rightarrow$ iii). Let $\rho_{0}, \lambda_{\rho}$ and $u_{\rho}$ be as in i) and let $\left(\rho_{h}\right)$ be a sequence in $\left.] 0,1\right]$ converging to 0 . We have $\left.\left.\rho_{h} \in\right] 0, \rho_{0}\right]$ eventually as $h \rightarrow \infty$. Then $\lambda_{\rho_{h}}$ and $v_{h}=u_{\rho_{h}} / \rho_{h}$ have the required properties.

Now we can give the main result in this section.

Theorem 3.20. - Let $\left(A 3^{+}\right)$hold and let $\lambda \in \mathbf{R}$ be essential for $\left(f_{0}+I_{M^{+}}\right)$.

Then $\lambda$ is an eigenvalue of (3.7) and of bifurcation for (3.2). More precisely, there exists $\rho_{0}>0,\left\{\lambda_{\rho}: 0<\rho \leq \rho_{0}\right\} \subset \mathbf{R},\left\{u_{\rho}: 0<\rho \leq\right.$ $\left.\rho_{0}\right\} \subset W$ such that

$$
\begin{gathered}
\left.\forall \rho \in] 0, \rho_{0}\right], \lambda_{\rho} L u_{\rho} \in \partial^{-} f\left(u_{\rho}\right) \text { and } \frac{1}{2}\left(L u_{\rho} \mid u_{\rho}\right)=\rho^{2} ; \\
\lim _{\rho \rightarrow 0^{+}}\left(\lambda_{\rho}, u_{\rho}\right)=(\lambda, 0), \lim _{\rho \rightarrow 0^{+}} f\left(u_{\rho}\right) / \rho^{2}=\lambda .
\end{gathered}
$$

Proof.-Let $\left(a_{k}\right)$ and $\left(b_{k}\right)$ be as in Definition 2.8. We want to apply Lemma 3.19 verifying the condition (3.19) $)_{\mathrm{iii}}$. Therefore let us consider $\varepsilon>0$ and a sequence $\left(\rho_{h}\right)$ in $\left.] 0,1\right]$ converging to 0 . Let us set $\rho_{\infty}=0$ and let $k \in \mathbf{N}$ be such that $\lambda-\varepsilon \leq a_{k}<b_{k} \leq \lambda+\varepsilon$.

By Propositions $3.4_{i}, 3.5_{i}, 3.15$, (A2) and Proposition 3.11, we can apply Theorem 2.17 to the sequence $\left(f_{\rho_{h}}\right)$ and the hypersurface $M^{+}$. Let $\left(\widehat{f}_{h}\right)$ and $\left(\tilde{f}_{h}\right)$ be the sequences given by Theorem 2.17 .

By $(2.17)_{\mathrm{i}} \quad \tilde{f}_{\infty}=\widehat{f}_{\infty}+I_{M^{+}}=f_{0}+I_{M^{+}}$. By $(2.17)_{\mathrm{viii}},(2.17)_{\mathrm{ix}}$, $(2.17)_{\mathrm{x}}$ and $(2.17)_{\mathrm{xi}}$ we can apply Theorem 2.16 to the sequence $\left(\widetilde{f}_{h}\right)$ with $a=a_{k}, b=b_{k}$. Let $h_{0} \in \mathbf{N}$ be such that $\forall h \geq h_{0}$, the pair $\left(\tilde{f}_{h}^{b_{k}}, \tilde{f}_{h}^{a_{k}}\right)$ is homotopically equivalent to the pair $\left(\left(f_{0}+I_{M^{+}}\right)^{b_{k}},\left(f_{0}+I_{M^{+}}\right)^{a_{k}}\right)$.

Then $\forall h \geq h_{0}, \widetilde{f}_{h}^{a_{k}}$ is not a weak deformation retract of $\tilde{f}_{h}^{b_{k}}$. Because of lower semicontinuity and equicoercivity, we can apply the deformation lemma 2.7 , obtaining that $\forall h \geq h_{0}, \exists u_{h} \in D\left(\widetilde{f}_{h}\right)$ such that $0 \in \partial^{-} \widetilde{f}_{h}\left(u_{h}\right), a_{k} \leq$ $\tilde{f}_{h}\left(u_{h}\right) \leq b_{k}$. In particular $u_{h} \in M^{+}$.

By $(2.17)_{\mathrm{iii}},(2.17)_{\mathrm{vi}}$ and $(2.18)$ we can apply Theorem 2.12, obtaining $\lambda_{h} L u_{h} \in \partial^{-} \hat{f}_{h}\left(u_{h}\right)$ for some $\lambda_{h} \in \mathbf{R}$. Since $\widehat{f}_{h}\left(u_{h}\right) \leq b_{k}$, by $(2.17)_{\text {vii }}$ we conclude that

$$
\begin{gathered}
\lambda_{h} L u_{h} \in \partial^{-} f_{\rho_{h}}\left(u_{h}\right), a_{k} \leq f_{\rho_{h}}\left(u_{h}\right) \leq b_{k} \\
-237-
\end{gathered}
$$




\section{Degiovanni}

eventually as $h \rightarrow+\infty$. Therefore condition (3.19) $)_{\mathrm{iii}}$ is fulfilled.

Finally, let us show that $\lambda$ is an eigenvalue of (3.7). Let $\rho_{0}, \lambda_{\rho}$ and $u_{\rho}$ be given by (3.19) . If we set $v_{\rho}=u_{\rho} / \rho$, we have $v_{\rho} \in M^{+}$and $\lambda_{\rho} L v_{\rho} \in$ $\partial^{-} f_{\rho}\left(v_{\rho}\right)$ by Proposition 3.4 $4_{\mathrm{iv}}$. By Proposition $3.15\left(v_{\rho_{h}^{\prime}}\right)$ converges to some $v \in M^{+}$along some sequence $\left(\rho_{h}^{\prime}\right)$ converging to zero. By Proposition 3.5 we conclude that

$$
\lambda L v \in \partial^{-} f_{0}(v) .
$$

Now we wish to consider two cases in which the condition that $\lambda$ is essential for $\left(f_{0}+I_{M^{+}}\right)$is not explicitely required.

The first one concerns the bifurcation from the "first eigenvalue" which has been studied, in a different framework, also in [29, 37]. A difference with respect to [37] is that in our situation the set $D(f)$ is not requested to be a cone. On the other hand in [29] a further assumption is imposed, in order to avoid the tangency between $D\left(f_{\rho}\right)$ and $M^{ \pm}$. In our approach we take advantage of the property (essentially proved in Theorem 2.17) taht, if $D\left(f_{\rho_{h}}\right)$ and $M^{ \pm}$are tangent at some $u_{h}$ and $\left(\rho_{h}\right) \rightarrow 0$, then $\left(f_{\rho_{h}}\left(u_{h}\right)\right) \rightarrow+\infty$.

THEOREM 3.21. - Let assumption $\left(A 3^{+}\right)$hold and let us suppose that $f_{0}(u)<+\infty$ for some $u$ with $(L u \mid u)>0$.

Then $\lambda:=\inf _{M^{+}} f_{0}$ is achieved, $\lambda$ is an eigenvalue of (3.7) and of bifurcation for (3.2). More precisely, all the thesis of Theorem 3.20 holds.

Proof.-By Proposition 3.6. iv we have that $D\left(f_{0}\right) \cap M^{+} \neq \emptyset$, so that $\lambda:=\inf _{H}\left(f_{0}+I_{M^{+}}\right)<+\infty$. By Proposition 3.16 we conclude that $\lambda \in \mathbf{R}$ and the infimum is achieved. Of course $\lambda$ is critical from below for $\left(f_{0}+I_{M^{+}}\right)$. By Proposition $3.12 \lambda$ is an eigenvalue of (3.7).

To conclude the proof, we want to apply Lemma 3.19 verifying the condition (3.19) $)_{\mathrm{ii}}$. Let $\left(\rho_{h}\right)$ be a sequence in $\left.] 0,1\right]$ converging to zero and let $\rho_{\infty}=0$. As in the proof of Theorem 2.10, we can apply Theorem 2.17 to the sequence $\left(f_{\rho_{h}}\right)$ and the hypersurface $M^{+}$, obtaining two sequences of functions $\left(\widehat{f}_{h}\right)$ and $\left(\tilde{f}_{h}\right)$. Of course $\lambda=\min _{H} \widetilde{f}_{\infty}$.

By $(2.17)_{x}$ it is readily seen that

$$
\min _{H} \tilde{f}_{\alpha} \geq \underset{h}{\limsup }\left(\inf _{H} \tilde{f}_{h}\right) .
$$

On the other hand, by (2.17) $)_{\text {viii }}$ and (2.17) $)_{\mathrm{xi}}$ the infima at the right hand side are achieved at some $u_{h} \in H$ with $0 \in \partial^{-} \tilde{f}_{h}\left(u_{h}\right)$. Again by $(2.17)_{x}$ and 
Bifurcation problems for nonlinear elliptic variational inequalities

$(2.17)_{\mathbf{x i}}$ we get

$$
\lim _{h} \tilde{f}_{h}\left(u_{h}\right)=\min _{H} \tilde{f}_{\infty}=\lambda .
$$

Now, as in the proof of Theorem 3.20, we conclude that $\lambda_{h} L u_{h} \in \partial^{-} \widehat{f}_{h}\left(u_{h}\right)$ for some $\lambda_{h} \in \mathbf{R}$ and that $\partial^{-} f_{\rho_{h}}\left(u_{h}\right)=\partial^{-} \widehat{f}_{h}\left(u_{h}\right), f_{\rho_{h}}\left(u_{h}\right)=\widehat{f}_{h}\left(u_{h}\right)$ eventually as $h \rightarrow \infty$.

In the second case, concerning the situation in which $f_{0}$ is a "quadratic form" in some generalized sense, we can give a bifurcation result also for higher eigenvalues of (3.7). We need a quite standard lemma on linear operators.

Lemma 3.22. - Let $X$ be a real Hilbert space and $B, K: X \rightarrow X$ two symmetric bounded operators such that $B K=K B$. Let $M=\{u \in X$ : $\left.\frac{1}{2}(B u \mid u)=1\right\}, \quad Q_{0}(u)=\frac{1}{2}(K u \mid u), Q(u)=\frac{1}{2}|u|^{2}-Q_{0}(u)$ and let us assume that $M$ is nonempty and sequentially weakly closed in $X, Q_{0 \mid M}$ is sequentially weakly continuous and

$$
\lim _{|u| \rightarrow+\infty} Q_{\mid M}(u)=+\infty,
$$

if $M$ is not bounded. Let $\lambda$ be a critical value of $Q_{\mid M}$.

Then there exists $\varepsilon_{0}>0$ such that:

i) $Q_{\mid M}$ has no critical values in $\left[\lambda-\varepsilon_{0}, \lambda+\varepsilon_{0}\right] \backslash\{\lambda\}$;

ii) for every $\left.\varepsilon \in] 0, \varepsilon_{0}\right]$, there does not exist any deformation

$$
\mathcal{H}:\left(Q_{\mid M}\right)^{\lambda+\varepsilon} \times[0,1] \rightarrow\left(Q_{\mid M}\right)^{\lambda+\varepsilon} \text { of }\left(Q_{\mid M}\right)^{\lambda+\varepsilon} \text { into }\left(Q_{\mid M}\right)^{\lambda-\varepsilon}
$$

iii) the linear space $\{u \in X: u-K u=\lambda B u\}$ has finite dimension.

Moreover if $\{u \in X: u-K u=\lambda B u\}$ has dimension one and $e$ is a critical point of $Q_{\mid M}$ with $Q(e)=\lambda$, then for every $\left.\left.\varepsilon \in\right] 0, \varepsilon_{0}\right]$ the set $\left(Q_{\mid M}\right)^{\lambda-\varepsilon} \cap\{u \in X:(u \mid e) \geq 0\}$ is not a weak retract of $\left(Q_{\mid M}\right)^{\lambda+\varepsilon} \cap\{u \in$ $X:(u \mid e) \geq 0\}$.

Proof. - Let $A=I-K$. Evidently $A B=B A$. First of all we claim that for every $x_{1}, x_{2} \in(\operatorname{ker} B)^{\perp}$ and for every $u_{1}, u_{2} \in X$,

$$
A u_{i}=B x_{i} \Rightarrow\left(x_{1} \mid u_{2}\right)=\left(x_{2} \mid u_{1}\right) \text {. }
$$

Infact, by the resolution of the identity associated with $B$, we can define a sequence of symmetric bounded linear operators $C_{h}: X \rightarrow X$ such that $B C_{h}=C_{h} B, A C_{h}=C_{h} A$ and

$$
\forall x \in(\operatorname{ker} B)^{\perp}, \lim _{h} C_{h} B x=x
$$




\section{Degiovanni}

in the weak topology of $X$.

In particular,

$$
\lim _{h}\left(C_{h} B x_{i} \mid u_{j}\right)=\left(x_{i} \mid u_{j}\right) .
$$

Since $\left(C_{h} B x_{1} \mid u_{2}\right)=\left(C_{h} A u_{1} \mid u_{2}\right)=\left(u_{1} \mid C_{h} A u_{2}\right)=\left(u_{1} \mid C_{h} B x_{2}\right)$, follows.

Let us remark that $(\operatorname{Ker} A) \cap(\operatorname{Ker} B)=\{0\}$. Infact, $\operatorname{Ker} B$ and $(\operatorname{Ker} B)^{\perp}$ are invariant for $A$. Let $u \in M \cap(\operatorname{Ker} B)^{\perp}$. If $v \in(\operatorname{Ker} A) \cap(\operatorname{Ker} B)$, we have $u+m v \in M$ and $Q(u+m v)=Q(u) \forall m \in \mathbf{N}$. Therefore the sequence $(u+m v)$ is bounded, hence $v=0$.

Now we claim that, if $\lambda_{1}, \lambda_{2} \in \mathbf{R}, u_{1}, u_{2} \in X, A u_{i}=\lambda_{i} B u_{i}$ and $\lambda_{1} \neq \lambda_{2}$, we have $\left(u_{1} \mid u_{2}\right)=0$.

Infact, let $u_{i}=v_{i}+w_{i}$ with $v_{i} \in \operatorname{ker} B, w_{i} \in(\operatorname{ker} B)^{\perp}$. Then $A v_{i}=$ $\lambda_{i} B v_{i}=0$, which implies $v_{i}=0$. By (3.23) we conclude that $\left(u_{1} \mid u_{2}\right)=0$.

Let $\wedge=\{\mu \in \mathbf{R}: A u=\mu B u$ for some $u \in X$ with $(B u \mid u)>0\}$ which is also the set of the critical values of $Q_{\mid M}$.

Let us remark that $\wedge \cap]-\infty, \lambda]$ is finite. Infact, by contradiction, if $\left(\lambda_{h}\right)$ is a sequence of distinct values in $\wedge \cap]-\infty, \lambda]$ and $u_{h} \in M, A u_{h}=\lambda_{h} B u_{h}$, we have $Q\left(u_{h}\right)=\lambda_{h} \leq \lambda$. Up to a subsequence, $\left(u_{h}\right)$ converges to some $u \in M$ in the weak topology of $X$ and $\left(Q\left(u_{h}\right)\right)$ converges to $Q(u)$. Therefore $\left(u_{h}\right)$ converges to $u$ in the strong topology of $X$. This is absurd, as $u \neq 0$ and the $u_{h}^{\prime} s$ are pairwise orthogonal.

Let $\wedge \cap]-\infty, \lambda]=\left\{\lambda_{1}, \ldots, \lambda_{h}\right\}$ with $\lambda_{1}<\ldots<\lambda_{h}=\lambda$ and let $V_{i}=\left\{u \in X: A u=\lambda_{i} B u\right\}$ for $i=1, \ldots, h$. Evidently $\lambda_{i}=\min _{h} Q$.

The subspaces $V_{i}$ are pairwise orthogonal. Moreover $V_{i} \cap M$ is sequentially weakly compact, hence bounded. An obvious adaptation of the proof of Proposition 3.17 shows that $V_{i}$ has finite dimension and $(B u \mid u)>0, \quad \forall u \in$ $V_{i} \backslash\{0\}$. Let

$$
V=V_{1} \oplus \ldots \oplus V_{h}, \quad Y=V^{\perp} .
$$

Since $B$ and $A$ leave invariant each $V_{i}$, the same property holds for $V$ and $Y$. Moreover $(B u \mid u)>0 \quad \forall u \in V \backslash\{0\}$.

Wé claim that $(A y \mid y) \geq \lambda_{1}(B y \mid y) \quad \forall y \in Y$. Infact, let $y \in Y$ and let $v \in V_{1} \cap M$. Without loss of generality, we can assume that $\frac{1}{2}(B y \mid y)<1$.

Let $s \in \mathbf{R}$ be such that $\frac{1}{2}(B y \mid y)+s^{2}=1$. Then $y+s v \in M$, so that

$$
\lambda_{1} \leq \frac{1}{2}(A(y+s v) \mid y+s v)=\frac{1}{2}(A y \mid y)+\lambda_{1}-\frac{\lambda_{1}}{2}(B y \mid y) .
$$


Bifurcation problems for nonlinear elliptic variational inequalities

Now let $\varepsilon_{0}$ be such that $\lambda_{h-1}<\lambda-\varepsilon_{0}<\lambda+\varepsilon_{0}<\min _{M \cap Y} Q$.

Evidently $Q_{\mid M}$ has no critical value in $\left[\lambda-\varepsilon_{0}, \lambda-\varepsilon_{0}\right] \backslash\{\lambda\}$.

Let $\left.\varepsilon \in] 0, \varepsilon_{0}\right]$.

Since $V$ has finite dimension, $M \cap V$ is not contractible in $X \backslash Y$. It is readily seen that

$$
M \cap V \subset\left(Q_{\mid M}\right)^{\lambda+\varepsilon} \subset X \backslash Y .
$$

Therefore $\left(Q_{\mid M}\right)^{\lambda+\varepsilon}$ is not contractible in $X \backslash Y$.

Let $e \in M \cap V_{h}$. It is easy to show that $X \backslash\{y+$ se $: y \in Y, s \geq 0\}$ is contractible in $X \backslash Y$. We claim that $\left(Q_{\mid M}\right)^{\lambda-\varepsilon} \subset X \backslash\{y+s e: y \in Y, s \geq 0\}$. Infact, let us assume that $y+s e \in M$. From $\frac{1}{2}(B y \mid y)+s^{2}=1$ we deduce that

$$
Q(y+s e)=\frac{1}{2}(A y \mid y)+\lambda_{h}-\frac{\lambda_{h}}{2}(B y \mid y) \geq \lambda_{h},
$$

since

$$
\begin{gathered}
\frac{1}{2}(A y \mid y)+\lambda_{h}-\frac{\lambda_{h}}{2}(B y \mid y) \geq \\
\geq \begin{cases}\lambda_{h}+\left(\lambda_{h+1}-\lambda_{h}\right) \frac{1}{2}(B y \mid y) & \text { if }(B y \mid y)>0 \\
\lambda_{h}+\left(\lambda_{1}-\lambda_{h}\right) \frac{1}{2}(B y \mid y) & \text { if }(B y \mid y) \leq 0\end{cases}
\end{gathered}
$$

Therefore there does not exist any deformation

$$
\mathcal{H}:\left(Q_{\mid M}\right)^{\lambda+\varepsilon} \times[0,1] \rightarrow X \backslash Y \text { of }\left(Q_{\mid M}\right)^{\lambda+\varepsilon} \text { into }\left(Q_{\mid M}\right)^{\lambda-\varepsilon} .
$$

Now let us assume that $V_{h}$ has dimension one and let $V^{\prime}=V_{1} \oplus \ldots \oplus V_{h-1}$.

Since $V^{\prime}$ has finite dimension, $M \cap V^{\prime}$ is not contractible in $\{u \in X$ : $(u \mid e) \geq 0\} \backslash\{y+s e: y \in Y, s \geq 0\}$.

On the other hand, $\forall u \in M \cap V^{\prime} Q(u) \leq \lambda_{h-1}$, so that

$$
\begin{aligned}
& M \cap V^{\prime} \subset\left(Q_{\mid M}\right)^{\lambda-\varepsilon} \cap\{u \in X:(u \mid e) \geq 0\} \subset \\
& \subset\{u \in X:(u \mid e) \geq 0\} \backslash\{y+s e: y \in Y, s \geq 0\} .
\end{aligned}
$$

In particular, $M \cap V^{\prime}$ is not contractible in $\left(Q_{\mid M}\right)^{\lambda-\varepsilon} \cap\{u \in X:(u \mid e) \geq 0\}$.

On the contrary,

$$
\mathcal{K}(u, t))(t e+(1-t) u) /\left[\frac{1}{2}(B(t e+(1-t) u) \mid t e+(1-t) u)\right]^{1 / 2}
$$


defines a contraction of $M \cap V^{\prime}$ in $\left(Q_{\mid M}\right)^{\lambda+\varepsilon} \cap\{u \in X:(u \mid e) \geq 0\}$.

Therefore $\left(Q_{\mid M}\right)^{\lambda+\varepsilon} \cap\{u \in X:(u \mid e) \geq 0\}$ is not a weak retract of $\left(Q_{\mid M}\right)^{\lambda+\varepsilon} \cap\{u \in X:(u \mid e) \geq 0\}$.

Let us denote by $\mathcal{C}$ the set of the $c^{\prime} s$ in $\mathbf{R}$ such that the function $\left\{u \longmapsto f_{0}(u)+\frac{c-\varepsilon}{2}|u|^{2}\right\}$ is convex for some $\varepsilon>0$ (in particular, $f_{0}(u)+\frac{c-\varepsilon}{2}|u|^{2} \geq 0 \forall u \in H$ by Proposition 3.6 $\left.6_{\mathrm{iii}}\right)$.

The set $\mathcal{C}$ is not empty. Infact $] 2 q,+\infty\left[\subset \mathcal{C}\right.$ by Proposition $3.6_{\mathrm{i}}$.

If $c \in \mathcal{C}$ and $u \in H$, let us denote by $i_{c}^{*} u$ the minimum point of the function $\left\{v \longmapsto f_{0}(v)+\frac{c}{2}|v|^{2}-(v \mid u)\right\}$. Evidently

$$
\begin{aligned}
& \forall u \in H, \quad\left(u-c i_{c}^{*} u\right) \in \partial^{-} f_{0}\left(i_{c}^{*} u\right) ; \\
& \forall u \in D\left(\partial^{-} f_{0}\right), \quad \forall \alpha \in \partial^{-} f_{0}(u), \quad u=i_{c}^{*}(\alpha+c u) .
\end{aligned}
$$

THEOREM 3.26. - Let $\left(A \Im^{+}\right)$hold and let us assume that

$$
\forall u, v \in H, \quad f_{0}(u+v)+f_{0}(u-v)=2 f_{0}(u)+2 f_{0}(v)
$$

and that

$$
c i_{c}^{*} L=L c i_{c}^{*}
$$

for some $c \in \mathcal{C}$.

Then every value $\lambda$, which is critical from below for $\lambda\left(f_{0}+I_{M^{+}}\right)$, is also essential for $\left(f_{0}+I_{M^{+}}\right)$. Moreover, $\left\{u \in H: \lambda L u \in \partial^{-} f_{0}(u)\right\}$ is a linear subspace of $H$ of finite dimension.

Proof. - Let $c \in \mathcal{C}$ be such that (3.28) holds. Combining Proposition $3.6_{\mathrm{i}}, 3.6_{\mathrm{iv}}$ and (3.27), we deduce that $D\left(f_{0}\right)$ is a linear subspace of $H$.

Moreover, we can define a scalar product $((\cdot \cdot))$ on $D\left(f_{0}\right)$ by $\forall u, v \in D\left(f_{0}\right), \quad((u \mid v))=\frac{1}{4}\left(2 f_{0}(u+v)+c|u+v|^{2}-2 f_{0}(u-v)-c|u-v|^{2}\right)$ so that the embedding $i:\left(D\left(f_{0}\right),\|\cdot\|\right) \rightarrow(H,|\cdot|)$ is continuous, where $\|\cdot\|$ is the norm associated with $((\cdot \cdot))$.

By the lower semicontinuity of $f_{0}$, the space $\left(D\left(f_{0}\right),((\cdot \cdot))\right)$ is complete, hence a Hilbert space. Moreover $i_{c}^{*}: H \rightarrow D\left(f_{0}\right)$ is the adjoint map of the embedding $i$. 
Bifurcation problems for nonlinear elliptic variational inequalities

Let us define two symmetric bounded linear operators $K, B: D\left(f_{0}\right) \rightarrow$ $D\left(f_{0}\right)$ by $K=c i_{c}^{*} i, B=i_{c}^{*} L i$. It is readily seen that

$$
\forall u \in D\left(f_{0}\right), \frac{1}{2}(((I-K) u \mid u))=f_{0}(u), \quad((B u \mid u))=(L u \mid u) .
$$

By (3.28) $B K=K B$.

If we set $M:=\left\{u \in D\left(f_{0}\right): \frac{1}{2}((B u \mid u))=1\right\}=M^{+} \cap D\left(f_{0}\right)$, $Q_{0}(u):=\frac{1}{2}((K u \mid u))=\frac{1}{2}|u|^{2}$, because of $\left(A 3^{+}\right) M$ is sequentially weakly closed in $D\left(f_{0}\right), Q_{0 \mid M}$ is sequentially weakly continuous and

$$
\lim _{\substack{\|u\| \rightarrow \infty \\ u \in M}} f_{0}(u)=+\infty
$$

if $M$ is not bounded.

Now we claim that for every $(\lambda, u)$ in $\mathbf{R} \times \mathbf{D}\left(\mathbf{f}_{\mathbf{0}}\right)$,

$$
\lambda L u \in \partial^{-} f_{0}(u) \Leftrightarrow u-K u=\lambda B u .
$$

Infact, by (3.24) and (3.25), $\lambda L u \in \partial^{-} f_{0}(u) \Leftrightarrow u=i_{c}^{*}(\lambda L u+c u) \Leftrightarrow$ $u-c i_{c}^{*} u=\lambda i_{c}^{*} u \Leftrightarrow u-K u=\lambda B u$.

By Proposition $3.12 \lambda \in \mathbf{R}$ is critical from below for $\left(f_{0}+I_{M^{+}}\right)$if and only if $\lambda$ is a critical value of $Q_{\mid M}$.

Moreover, the norm $\|\cdot\|$ and the graph metric $d^{*}$ associated with $f_{0}$ (see (2.5)) induce on $D\left(f_{0}\right)$ the same topology and $\forall b \in \mathbf{R},\left(f_{0}+I_{M^{+}}\right)^{b}=$ $\left(Q_{\mid M}\right)^{b}$.

Applying Lemma 3.22, the thesis follows.

THEOREM 3.29.- - Let us assume that $\left(A 3^{+}\right),(3.27)$ and (3.28) hold and let $\lambda \in \mathbf{R}$ be an eigenvalue of (3.7).

Then $\lambda$ is of bifurcation for (3.2). More precisely, all the thesis of Theorem 3.20 holds.

Proof.- It is sufficient to combine Proposition 3.12 and Theorem 3.26 with Theorem 3.20.

In the case in which the function $f$ is smooth, several multiplicity results are known for bifurcation (see $[7,21,26,34,35]$ ). Here we give a first result for simple eigenvalues. 


\section{Degiovanni}

Theorem 3.30.- Let us assume that $\left(\mathrm{AS}^{+}\right)$, (3.27) and (3.28) hold and let $\lambda \in \mathbf{R}, e \in M^{+}$be such that $\lambda L e \in \partial^{-} f_{0}(e)$. Let us suppose that $\left\{u \in H: \lambda L u \in \partial^{-} f_{0}(u)\right\}$, which is a linear space by Theorem 3.26, has dimension one.

Then there exist $\rho_{0}>0,\left\{\lambda_{\rho}^{(i)}: 0<\rho \leq \rho_{0}, i=1,2\right\} \subset \mathbf{R},\left\{u_{\rho}^{(i)}: 0 \rho \leq \rho_{0}\right.$, $i=1,2\} \subset W$ such that

$$
\begin{gathered}
\left.\forall \rho \in] 0, \rho_{0}\right], \quad \forall i=1,2, \lambda_{\rho}^{(i)} L u_{\rho}^{(i)} \in \partial^{-} f\left(u_{\rho}^{(i)}\right) \\
\text { and } \quad \frac{1}{2}\left(L u_{\rho}^{(i)} \mid u_{\rho}^{(i)}\right)=\rho^{2} \\
\lim _{\rho \rightarrow 0^{+}}\left(\lambda_{\rho}^{(i)}, u_{\rho}^{(i)}\right)=(\lambda, 0), \quad \lim _{\rho \rightarrow 0^{+}} f\left(u_{\rho}^{(i)}\right) / \rho^{2}=\lambda \\
\lim _{\rho \rightarrow 0^{+}} u_{\rho}^{(1)} / \rho=-e, \lim _{\rho \rightarrow 0^{+}} u_{\rho}^{(2)} / \rho=-e .
\end{gathered}
$$

Proof. - First of all it is sufficient to show that there exist $\rho_{0}>0$, $\left\{\lambda_{\rho}: 0<\rho \leq \rho_{0}\right\} \subset \mathbf{R}, \quad\left\{u_{\rho}: 0<\rho \leq \rho_{0}\right\} \subset W$ such that

$$
\begin{gathered}
\left.\forall \rho \in] 0, \rho_{0}\right], \lambda_{\rho} L u_{\rho} \in \partial^{-} f\left(u_{\rho}\right) \text { and } \frac{1}{2}\left(L u_{\rho} \mid u_{\rho}\right)=\rho^{2} ; \\
\lim _{\rho \rightarrow 0^{+}}\left(\lambda_{\rho}, u_{\rho}\right)=(\lambda, 0), \lim _{\rho \rightarrow 0^{+}} f\left(u_{\rho}\right) / \rho^{2}=\lambda ; \\
\lim _{\rho \rightarrow 0^{+}} u_{\rho} / \rho=e .
\end{gathered}
$$

Let $\Sigma=\{u \in H:(u \mid e) \geq 0\}, \quad \partial \Sigma=\{u \in H:(u \mid e)=0\}$ and let $g=f+I_{\Sigma}: \bar{W} \rightarrow \mathbf{R} \cup\{+\infty\}$.

We want to apply Theorem 3.20 with the function $f$ substituted by $g$. It is readily seen that $g(0)=0,0 \in \partial^{-} g(0), g$ is lower semicontinuous and the function $\left.\left\{u \longmapsto g(u)+q|u|^{2}\right)\right\}$ is convex. Moreover, if we define $g_{\rho}$ according to the assumption (A2) and we set $g_{0}=f_{0}+I_{\Sigma}$, we claim that

$$
g_{0}=\Gamma^{-}(h) \lim _{h} g_{\rho_{h}}
$$

for every sequence $\left(\rho_{h}\right)$ in ]0,1] converging to zero.

Infact, since $\Sigma$ is closed, by (A2) we have only to verify that for every $u \in D\left(f_{0}\right) \cap \Sigma$ there exists a sequence $\left(u_{h}\right)$ in $\Sigma$ converging to $u$ with

$$
\underset{h}{\limsup } f_{\rho_{h}}\left(u_{h}\right) \leq f_{0}(u) .
$$


Bifurcation problems for nonlinear elliptic variational inequalities

Let $\left(w_{h}\right)$ be a sequence converging to $u$ with $\left(f_{\rho_{h}}\left(w_{h}\right)\right)$ converging to $f_{0}(u)$. If $w_{h} \in \Sigma$, let us set $u_{h}=w_{h}$. Otherwise, let $u_{h} \in \partial \Sigma$ be defined by

$$
\left.u_{h}=\left(|e|^{2} w_{h}-\left(w_{h} \mid e\right) e\right) /|e|^{2}-\left(w_{h} \mid e\right)\right) .
$$

It is readily seen that $\left(u_{h}\right)$ converges to $u$ and, by Proposition 3.4

$$
\underset{h}{\limsup } f_{\rho_{h}}\left(u_{h}\right) \leq f_{0}(u) .
$$

Finally, since $g \geq f$, condition $\left(\mathrm{A}^{+}\right)$is satisfied by $g$.

Let $((\cdot \cdot))$ be the scalar product on $D\left(f_{0}\right)$ and $K, B: D\left(f_{0}\right) \rightarrow D\left(f_{0}\right)$ the symmetric bounded linear operators defined in the proof of Theorem 3.26. We know that $\lambda$ is a critical value of $f_{0}$ on the submanifold $M$ of $D\left(f_{0}\right)$. Let again $\varepsilon_{0}$ be given by Lemma 3.22.

It is readily seen that $(I-K) i_{c}^{*} e=\lambda B i_{c}^{*} e$. Therefore $\|e\|^{2} i_{c}^{*} e=|e|^{2} e$. In particular,

$$
\left\{u \in D\left(f_{0}\right):((u \mid e)) \geq 0\right\}=D\left(f_{0}\right) \cap \Sigma .
$$

Then for every $b \in \mathbf{R}$,

$$
\left(g_{0}+I_{M^{+}}\right)^{b}=\left(f_{0 \mid M}\right)^{b} \cap \Sigma,
$$

Moreover the norm $\|\cdot\|$ and the metric $d^{*}$ associated with $g_{0}$ induce on $D\left(g_{0}\right)$ the same topology. By Lemma 3.22, $\lambda$ is essential for $\left(g_{0}+I_{M^{+}}\right)$.

Let $\rho_{0}, \lambda_{\rho}$ and $u_{\rho}$ be obtained by applying Theorem 3.20 to the function g. By $\left(\mathrm{A}^{+}\right)$, along some sequence $\left(\rho_{h}\right)$ conveging to zero we have that $\left(u_{\rho} / \rho\right)$ converges to some $v \in M^{+}$. Since $\partial^{-} g$ and $\partial^{-} f$ agree on $\stackrel{\circ}{\Sigma}$, the proof will be conclued if we show that $v=e$.

Let us argue by contradiction. By Propositions 3.4 iv and 3.5, $\lambda L v \in$ $\partial^{-} g_{0}(v)=\partial^{-}\left(f_{0}+I_{\Sigma}\right)(v)$. Since $v \neq e$ it must be $v \in \partial \Sigma$. A fortiori $\lambda L v \in \partial^{-}\left(f_{0}+I_{\partial \Sigma}\right)(v)$. Since $e$ and $-e$ belong to $D\left(f_{0}\right)$, by Remark 2.11 $D\left(f_{0}\right)$ and $\partial \Sigma$ are not tangent at $v$.

Therefore, by Theorem $2.12 \lambda L v-\mu e \in \partial^{-} f_{0}(v)$ for some $\mu \in \mathbf{R}$. Since $v \neq e$, it is $\mu \neq 0$. Then

By (3.25) we have $v=i_{c}^{*}(\lambda L v-\mu e+c v)$, namely $(I-K) v=\lambda B v-\mu i_{c}^{*} e$.

$$
\begin{gathered}
\mu|e|^{2}=\lambda((B v \mid e))-(((I-K) v \mid e))= \\
=\lambda((v \mid B e))-((v \mid(I-K) e))=0 \\
-245-
\end{gathered}
$$




\section{Degiovanni}

hence $\mu=0$, which is a contradiction.

\section{Bifurcation for elliptic variational inequalities}

We want to show an application of the results of the previous section to elliptic variational inequalities of the form

$$
\left\{\begin{array}{c}
(\lambda, u) \in \mathbf{R} \times\left(\mathbf{K} \cap L^{\infty}(\Omega)\right) \\
\int_{\Omega}\left(\sum_{i, j} a_{i j} D_{i} u D_{j}(v-u)+a_{0} u(v-u)+g(x, u)(v-u)\right) d x \geq \\
\geq \lambda \int_{\Omega} u(v-u) d x \quad \forall v \in \mathbf{K}
\end{array}\right.
$$

More precisely, let $\Omega$ be a bounded open subset of $\mathbf{R}^{n}$ and let us assume that

(H1) for $i, j=1, \ldots, n, a_{i j} \in L^{\infty}(\Omega), a_{i j}=a_{j i}$ and there exists $\nu>0$ such that

$$
\forall \xi \in \mathbf{R}^{n}, \quad \sum_{i, j} a_{i j} \xi_{i} \xi_{j} \geq \nu \sum_{i} \xi_{i}^{2}
$$

(H2) $\quad a_{0} \in L^{p}(\Omega)$ for some $p>n / 2$ if $n \geq 2, a_{0} \in L^{1}(\Omega)$ if $n=1$;

(H3) $g: \Omega \times \mathbf{R} \rightarrow \mathbf{R}$ is a Caratheodory function such that for almost every $x \in \Omega$

$$
\lim _{s \rightarrow 0} \frac{g(x, s)}{s}=0
$$

and such that

$$
\begin{gathered}
|g(x, s)| \leq a_{1}(x) \eta(s)|s|, \\
\left(g\left(x, s_{1}\right)-g\left(x, s_{2}\right)\right)\left(s_{1}-s_{2}\right) \geq \\
\geq-a_{1}(x)\left(\eta\left(s_{1}\right)+\eta\left(s_{2}\right)\right)\left(s_{1}-s_{2}\right)^{2}
\end{gathered}
$$

where $\eta: \mathbf{R} \rightarrow \mathbf{R}^{+}$is continuous and $a_{1} \in L^{p}(\Omega)$ for some $p>n / 2$ if $n \geq 2$, $a_{1} \in L^{1}(\Omega)$ if $n=1$;

(H4) $\quad \mathbf{K}$ is the set

$\left\{u \in W_{0}^{1,2}(\Omega): \varphi_{1}(x) \leq \widetilde{u}(x) \leq \varphi_{2}(x)\right.$ quasi-everywhere in $\left.\Omega\right\}$

where $\varphi_{1}: \Omega \longrightarrow[-\infty, 0]$ is quasi-upper semicontinuous, $\varphi_{2}: \Omega \longrightarrow[0,+\infty]$ is quasi-lower semicontinuous and for every $u$ in $W_{0}^{1,2}(\Omega)$, tildeu $: \Omega \longrightarrow \mathbf{R}$ is the quasi-continuous function defined quasi-everywhere by

$$
\widetilde{u}(X)=\lim _{\rho \rightarrow 0^{+}} m(B(x, \rho))^{-1} \int_{B-x, \rho)} u(y) d y .
$$


(4.2) Remark. - Some comments are in order :

i) If $g: \bar{\Omega} \times \mathbf{R} \longrightarrow \mathbf{R}$ is of class $C^{1}$ and $g(x, 0)=0, D_{s} g(x, 0)=0$, then hypothesis (H3) is automatically satisfied;

ii) If $\varphi_{1}: \Omega \longrightarrow[-\infty, 0]$ and $\varphi_{2}: \Omega \longrightarrow[0,+\infty]$ are two Borel functions, then (see [2, Theorem 3.2]) there exist a quasi-upper semicontinuous function $\widehat{\varphi}_{1}: \Omega \longrightarrow[-\infty, 0]$ and a quasi-lower semicontinuous function $\hat{\varphi}_{2}: \Omega \longrightarrow[0,+\infty]$ such that

$\left\{u \in W_{0}^{1,2}(\Omega): \widehat{\varphi_{1}}(x) \leq \widetilde{u}(x) \leq \widehat{\varphi}_{2}(x)\right.$ quasi-everywhere in $\left.\Omega\right\}=$ $=\left\{u \in W_{0}^{1,2}(\Omega): \varphi_{1}(x) \leq \widetilde{u}(x) \leq \varphi_{2}(x)\right.$ quasi-everywhere in $\left.\Omega\right\}$.

Our aim is to study the pairs $(\lambda, u)$ satisfying (4.1). Because of (H3), $g(x, 0)=0$. Therefore for every $\lambda$ in $\mathbf{R}$, the pair $(\lambda, 0)$ verifies (4.1).

(4.3) Definition A real number $\lambda$ is said to be of bifurcation for (4.1), if there exists a sequence $\left(\left(\lambda_{h}, u_{h}\right)\right)$ of solutions of $(4.1)$ with $u_{h} \neq 0$ and

$$
\lim _{h} \lambda_{h}=\lambda, \quad \lim _{h} u_{h}=0 \quad \text { in } \quad L^{\infty}(\Omega) .
$$

Let $\zeta: \mathbf{R} \rightarrow[-2,2]$ be a smooth increasing function such that $\left|\zeta^{\prime}(s)\right| \leq$ $1 \forall s \in \mathbf{R}$ and $\zeta(s)=s \forall s \in[-1,1]$. We set $\widetilde{g}(x, s)=g(x, \zeta(s)), \widetilde{G}(x, s)=$ $\int_{0}^{s} \tilde{g}(x, \tau) d \tau$.

Then $\tilde{g}$ satisfies the hypothesis (H3) with the function $\eta$ substituted by the constant $\bar{\eta}=\max \{\eta(s):-2 \leq s \leq 2\}$.

Let $(H,(\cdot \mid \cdot))$ denote the space $L^{2}(\Omega)$ endowed with the usual scalar product and let $f, f_{0}: H \rightarrow \mathbf{R} \cup\{+\infty\}$ be defined by

$$
\begin{gathered}
f(u)= \begin{cases}\int_{\Omega}\left(\frac{1}{2} \sum_{i, j} a_{i j} D_{i} u D_{j} u+\frac{1}{2} a_{0} u^{2}+\widetilde{G}(x, u)\right) d x, & u \in \mathbf{K} \\
+\infty & u \in H \backslash \mathbf{K}\end{cases} \\
f_{0}(u)= \begin{cases}\int_{\Omega}\left(\frac{1}{2} \sum_{i, j} a_{i j} D_{i} u D_{j} u+\frac{1}{2} a_{0} u^{2}\right) d x, & u \in \mathbf{K}_{0} \\
+\infty & u \in H \backslash \mathbf{K}_{0}\end{cases}
\end{gathered}
$$

where $K_{0}$ is the closure in $W_{0}^{1,2}(\Omega)$ of the set $\left(U_{t>0} t \mathbf{K}\right)$.

Evidently, $\mathbf{K}_{0}$ is a closed convex cone.

Finally, let us set $f_{\rho}(u)=\rho^{-2} f(\rho u)$ for $\rho>0, u \in H$.

Lemma 4.4.- Let $X$ be a normed space, $g: X \rightarrow \mathbf{R}$ a continuous convex function and $K$ a closed convex subset of $X$ with $0 \in K$. Let $K_{0}$ 
denote the closure of $\left(\cup_{t>0} t K\right)$. Then for every sequence $\left(\rho_{h}\right)$ in $] 0,+\infty \mid$ conerging to zero, we have

$$
\left(g+I_{K_{0}}\right)=\Gamma^{-}\left(X_{w}\right) \lim _{h}\left(g+I_{\rho_{h}^{-1} K}\right)
$$

where $X_{w}$ denotes the space $X$ endowed with the weak topology.

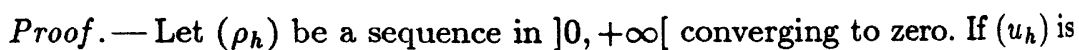
a sequence in $X$ weakly converging to $u$ and $\left(g+I_{\rho_{h}^{-1} K}\right)\left(u_{h}\right) \leq c \in \mathbf{R}$, we have $u_{h} \in \rho_{h}^{-1} K$ and $g\left(u_{h}\right) \leq c$.

Therefore $u \in K_{0}$ and $\left(g+I_{K_{0}}\right)(u)=g(u) \leq c$.

Now let $u \in X$. We have to prove that there exists a sequence $\left(u_{h}\right)$ weakly converging to $u$ with

$$
\left(g+I_{K_{0}}\right)(u) \geq \underset{h}{\limsup }\left(g+I_{\rho_{h}^{-1} K}\right)\left(u_{h}\right)
$$

To this aim, it is enough to show that for every $u \in K_{0}$, for every $r, \varepsilon>0$, there exists $h_{0} \in \mathbf{N}$ such that for every $h \geq h_{0}$,

$$
\inf \left\{g(v): v \in\left(\rho_{h}^{-1} K\right) \cap B(u, r)\right\}<g(u)+\varepsilon
$$

This last fact is obvious, as we can find $v \in \bigcup_{t>0} t K$ such that $v \in$ $B(u, r), g(v)<g(u)+\varepsilon$.

Proposition 4.5. - The following facts hold:

i) $f$ is lower semicontinuous and the function $\left\{u \mapsto f(u)+q|u|^{2}\right\}$ is convex for some $q \in \mathbf{R}^{+}$;

ii) for every sequence $\left(\rho_{h}\right)$ in $] 0,+\infty[$ converging to zero, we have

$$
f_{0}=\Gamma^{-}(H) \lim _{h} f_{\rho_{h}}
$$

iii) for every sequence $\left(u_{h}\right)$ in $H \backslash\{0\}$ with

$$
\sup _{h}\left|u_{h}\right|^{-2} f\left(u_{h}\right)<+\infty
$$

the sequence $\left(u_{h} /\left|u_{h}\right|\right)$ has a convergent subsequence; 
Bifurcation problems for nonlinear elliptic variational inequalities

iv) for every $\lambda \in \mathbf{R}, v \in \mathbf{K}$, we have $\lambda u \in \partial^{-} f(u)$ if and only if

$$
\begin{gathered}
\int_{\Omega}\left(\sum_{i, j} a_{i j} D_{i} u D_{j}(v-u)+a_{0} u(v-u)+\widetilde{g}(x, u)(v-u)\right) d x \geq \\
\geq \lambda \int_{\Omega} u(v-u) d x, \quad \forall v \in \mathbf{K} ;
\end{gathered}
$$

v) for every $\lambda \in \mathbf{R}, u \in \mathbf{K}_{0}$, we have $\lambda u \in \partial^{-} f_{0}(u)$ if and only if

$$
\begin{array}{ll}
\int_{\Omega}\left(\sum_{i, j} a_{i j} D_{i} u D_{j}(v-u)+a_{0} u(v-u)\right) d x \geq \\
\geq \lambda \int_{\Omega} u(v-u) d x, \quad \forall v \in \mathbf{K}_{0} .
\end{array}
$$

Proof.-By (H1), (H2) and (H3), we have for every $u \in W_{0}^{1,2}(\Omega)$,

$$
\begin{aligned}
f(u) & \geq \frac{1}{2} \int_{\Omega}\left(v|d u|^{2}+a_{0} u^{2}+a_{1} \bar{\eta} u^{2}\right) d x \geq \\
& \geq \frac{v}{4} \int_{\Omega}|D u|^{2} d x-\text { const. } \int_{\Omega} u^{2} d x
\end{aligned}
$$

Therefore, in order to get the lower semicontinuity of $f$ in the strong topology of $L^{2}(\Omega)$, it is sufficient to prove the sequential lower semicontinuity of $f$ in the weak topology of $W_{0}^{1,2}(\Omega)$.

For every $u$ in $\mathbf{K}$ we have

$$
\begin{gathered}
f(u)=\int_{\Omega} \frac{1}{2} \sum_{i, j} a_{i j} D_{i} u D_{j} u d x+\int_{\Omega}\left(\widetilde{G}(x, u)+\frac{1}{2} a_{1} \bar{\eta} u^{2} b i g\right) d x+ \\
+\frac{1}{2} \int_{\Omega}\left(a_{0}-a_{1} \bar{\eta}\right) u^{2} d x
\end{gathered}
$$

Now the first two terms are continuous in the strong topology of $W_{0}^{1,2}(\Omega)$ and convex. The third term is sequentially continuous in the weak topology of $W_{0}^{1,2}(\Omega)$. We deduce that $f$ is sequentially weakly lower semicontinuous in $W_{0}^{1,2}(\Omega)$. Moreover, it is readily seen that the function $\left\{u \mapsto f(u)+q|u|_{L^{2}}^{2}\right\}$ is convex for some $q \in \mathbf{R}^{+}$.

Let us prove ii). Let $\left(\rho_{h}\right)$ be a sequence in. $] 0,+\infty$ [ converging to zero. $\mathrm{By}$ (4.6) it is enough to prove the $\Gamma$-convergence in the weak topology of $W_{0}^{1,2}(\Omega)$. 


\section{Degiovanni}

Because of (H2) and (H3), we have

$$
\lim _{h}\left(\frac{1}{2} \int_{\Omega} a_{0} u_{h}^{2} d x+\rho_{h}^{-2} \int_{\Omega} \widetilde{G}\left(x, \rho_{h} u_{h}\right) d x\right)=\frac{1}{2} \int_{\Omega} a_{0} u^{2} d x
$$

whenever $\left(u_{h}\right)$ is a sequence weakly converging in $W_{0}^{1,2}(\Omega)$ to $u$. Then property ii) follows from Lemma 4.4.

Property iii) is an immediate consequence of Rellich's theorem. Infact, if $v_{h}=u_{h} /\left|u_{h}\right|$, we have by (4.6)

$$
\begin{gathered}
\frac{v}{4} \int_{\Omega}\left|D v_{h}\right|^{2} d x-\text { const. }= \\
=\left|u_{h}\right|^{-2}\left(\frac{v}{4} \int_{\Omega}\left|D u_{h}\right|^{2} d x-\text { const. } \int_{\Omega} u_{h}^{2} d x\right) \leq\left|u_{h}\right|^{-2} f\left(u_{h}\right) .
\end{gathered}
$$

To prove iv), it is sufficient to see that for every $u \in \mathbf{K}, \alpha \in H$, we have $\alpha \in \partial^{-} f(u)$ if and only if

$$
\begin{array}{cc}
\int_{\Omega}\left(\sum_{i, j} a_{i j} D_{i} u D_{j}(v-u)+a_{0} u(v-u)+\widetilde{g}(x, u)(v-u)\right) d x \geq \\
\geq \int_{\Omega} \alpha(v-u) d x, \quad \forall v \in \mathbf{K} .
\end{array}
$$

Since the function $\left\{u \mapsto q|u|^{2}\right\}$ is Fréchet differentiable in $L^{2}(\Omega)$, we have

$$
\partial^{-}\left(f+q|\cdot|^{2}\right)(u)=\partial^{-} f(u)+2 q u .
$$

Taking into account the convexity of $\left\{u \mapsto f(u)+q|u|^{2}\right\}$, we conclude that $\alpha \in \partial^{-} f(u)$ if and only if

$$
\begin{gathered}
\lim _{t \rightarrow 0^{+}} \frac{f(u+t(v-u))+q|u+t(v-u)|^{2}-f(u)-q|u|^{2}}{t} \geq \\
\geq(\alpha+2 q u \mid v-u) \quad \forall v \in \mathbf{K},
\end{gathered}
$$

which amounts to stating iv).

The proof of $v$ ) is similar.

According to the abstract theory of section 3, we shall consider

$$
\left\{\begin{array}{c}
(\lambda, u) \in \mathbf{R} \times \mathbf{K}_{0} \\
\int_{\Omega}\left(\sum_{i, j} a_{i j} D_{i} u D_{j}(v-u)+a_{0} u(v-u)\right) d x \geq \\
\geq \lambda \Omega u(v-u) d x \quad \forall v \in \mathbf{K}_{0} \\
-250-
\end{array}\right.
$$


as the "linearized" problem associated with (4.1).

DEFINITION 4.8. - A real number $\lambda$ is said to be an eigenvalue of (4.7), if the pair $(\lambda, u)$ satisfies (4.7) for some $u \neq 0$.

Proposition 4.9. - Let $u \in W_{0}^{1,2}(\Omega)$. Then $u \in \mathbf{K}_{0}$ if and only if $\tilde{u}(x) \geq 0$ quasi-everywhere in $\varphi_{1}^{-1}(0)$ and $\tilde{u}(x) \leq 0$ quasi-everywhere in $\varphi_{2}^{-1}(0)$. Moreover $\mathbf{K}_{0}=\{0\}$ if and only if $\varphi_{1}$ and $\varphi_{2}$ are zero quasieverywhere in $\Omega$.

Proof. - Let $u \in \mathbf{K}_{0}$ and let $\left(u_{h}\right)$ be a sequence in $\bigcup_{t>0} t \mathbf{K}$ converging to $u$ in the strong topology of $W_{0}^{1,2}(\Omega)$. Of course $\widetilde{u}_{h}(x) \geq 0$ quasi-everywhere in $\varphi_{1}^{-1}(0)$ and $\tilde{u}_{h}(x) \leq 0$ quasi-everywhere in $\varphi_{2}^{-1}(0)$. Up to a subsequence, $\left(\tilde{u}_{h}\right)$ converges to $\widetilde{u}$ pointwise quasi-everywhere, hence the thesis.

Let us prove the converse. To see that $u \in \mathbf{K}_{0}$, it is enough to show that $u^{+}$and $-u^{-}$belong to $\mathbf{K}_{0}$. Let us consider $-u^{-}$, the argument for $u^{+}$being similar. Since $\widetilde{u}^{-}(x)=0$ quasi-everywhere in $\varphi_{1}^{-1}(0),\left(\max \left\{h \varphi_{1},-\tilde{u}^{-}\right\}\right)$is a decreasing sequence of nonpositive quasi-upper semicontinuous functions converging to $-\widetilde{u}^{-}$pointwise quasi-everywhere. By [10, Lemma 1.6] there exists a sequence $\left(v_{h}\right)$ in $W_{0}^{1,2}(\Omega)$ converging to $-u^{-}$in the strong topology of $W_{0}^{1,2}(\Omega)$ with $\widetilde{v}_{h} \geq h \varphi_{1}$ quasi-everywhere in $\Omega$. Without loss of generality, we can assume $v_{h} \leq 0$. Since $v_{h} \in \bigcup_{t>0} t \mathbf{K}$, the thesis follows.

It is clear that $\mathbf{K}_{0}=\{0\}$ if $\varphi_{1}$ and $\varphi_{2}$ are zero quasi-everywhere. Let us suppose that $\mathbf{K}_{0}=\{0\}$. By[10, Lemma 1.5], there exists a decreasing sequence $\left(w_{h}\right)$ in $W_{0}^{1,2}(\Omega)$ such that $\left(\widetilde{w}_{h}\right)$ converges to $\varphi_{1}$ pointwise quasieverywhere. Without loss of generality we can suppose $w_{h} \leq 0$. Since $w_{h} \in \mathbf{K} \subset \mathbf{K}_{0}$, we have $w_{h}=0$, so that $\varphi_{1}(x)=0$ quasi-everywhere. In an analogous way we can see that $\varphi_{2}(x)=0$ quasi-everywhere in $\Omega$.

THEOREM 4.10. - Let $\lambda$ be a real number. If $\lambda$ is of bifurcation for (4.1), then $\lambda$ is an eigenvalue of (4.7).

Proof. - Let $\left(\left(\lambda_{h}, u_{h}\right)\right)$ be a sequence as in Definition 4.3. Then $\left(u_{h}\right)$ converges to zero also in $L^{2}(\Omega)$ and by Proposition $4.5_{\mathrm{iv}}$ we have $\lambda_{h} u_{h} \in$ $\partial^{-} f\left(u_{h}\right)$ eventually as $h \rightarrow \infty$. Combining Proposition 4.5 with Theorem 3.14 (with $L$ equal to the identity map), the thesis follows.

Definition 4.11. - An eigenvalue $\lambda$ of (4.7) is said to be essential, if 
there exists two sequences $\left(a_{k}\right)$ in $]-\infty, \lambda\left[\right.$ and $\left(b_{k}\right)$ in $] \lambda,+\infty[$ converging to $\lambda$ such that

i) for every $k \in \mathbf{N}, a_{k}$ and $b_{k}$ are not eigenvalues of (4.7);

ii) for every $k \in \mathbf{N}$, the set

$$
\left\{u \in \mathbf{K}_{0}: \frac{1}{2} \int_{\Omega} u^{2} d x=1, \frac{1}{2} \int_{\Omega}\left(\sum_{i, j} a_{i j} D_{i} u D_{j} u+a_{0} u^{2}\right) d x \leq a_{k}\right\}
$$

is not a weak deformation retract of the set

$$
\left\{u \in \mathbf{K}_{0}: \frac{1}{2} \int_{\Omega} u^{2} d x=1, \frac{1}{2} \int_{\Omega}\left(\sum_{i, j} a_{i j} D_{i} u D_{j} u+a_{0} u^{2}\right) d x \leq b_{k}\right\}
$$

endowed with the strong topology of $W_{0}^{1,2}(\Omega)$.

LEMMA 4.12. - There exists a continuous function $c: \mathbf{R} \rightarrow \mathbf{R}^{+}$such that for every $(\lambda, u)$ in $\mathbf{R} \times \mathbf{K}$ satisfying

$$
\begin{array}{cc}
\int_{\Omega}\left(\sum_{i, j} a_{i j} D_{i} u D_{j}(v-u)+a_{0} u(v-u)+\widetilde{g}(x, u)(v-u)\right) d x \geq \\
\geq \lambda \int_{\Omega} u(v-u) d x, & \forall v \in \mathbf{K},
\end{array}
$$

we have $u \in L^{\infty}(\Omega)$ and

$$
\|u\|_{L^{\infty}(\Omega)} \leq c(\lambda)\|u\|_{L^{2}(\Omega)} .
$$

Proof.- It is sufficient to remark that, if we set

$$
u_{k}= \begin{cases}u-k & \text { for } u>k \\ 0 & \text { for }|u| \leq k \\ u+k & \text { for } u<-k\end{cases}
$$

then the function $u-u_{k}$ belongs to $\mathbf{K}$, so that

$$
\int_{\Omega}\left(\sum_{i, j} a_{i j} D_{i} u D_{j} u_{k}+a_{0} u u_{k}+\widetilde{g}(x, u) u_{k}\right) d x \leq \lambda \int_{\Omega} u u_{k} d x
$$

Since $|g(x, u)| \leq a_{1}(x) \bar{\eta}|u|$, the argument of [39, Theorem 4.1 and Remark $4.2]$ works also in this case. 
Bifurcation problems for nonlinear elliptic variational inequalities

THEOREM 4.13. - Let $\lambda$ be a real number. If $\lambda$ is an essential eigenvalue of (4.7), then $\lambda$ is of bifurcation for (4.1). More precisely, there exist $\rho_{0}>0,\left\{\lambda_{\rho}: 0<\rho \leq \rho_{0}\right\} \subset \mathbf{R},\left\{u_{\rho}: 0<\rho \leq \rho_{0}\right\} \subset \mathbf{K}$ such that $\left(\lambda_{\rho}, u_{\rho}\right)$ satisfies (4.1), $\frac{1}{2} \int_{\Omega} u_{\rho}^{2} d x=\rho^{2}$ and

$$
\begin{gathered}
\lim _{\rho \rightarrow 0^{+}} u_{\rho}=0 \text { in } L^{\infty} \text { and in } W_{0}^{1,2}(\Omega), \\
\lim _{\rho \rightarrow 0^{+}} \lambda_{\rho}=\lim _{\rho \rightarrow 0^{+}} \frac{1}{2 \rho^{2}} \int_{\Omega}\left(\sum_{i, j} a_{i j} D_{i} u_{\rho} D_{j} u_{\rho}+a_{0} u_{\rho}^{2}\right) d x=\lambda .
\end{gathered}
$$

Proof.- Let $M=\left\{u \in L^{2}(\Omega): \frac{1}{2} \int_{\Omega} u^{2} d x=1\right\}$. It is readily seen that $D\left(f_{0}+I_{M}\right) \subset W_{0}^{1,2}(\Omega)$ and the graph metric $d^{*}$ associated with $\left(f_{0}+I_{M}\right)$ induces on $D\left(f_{0}+I_{M}\right)$ the strong topology of $W_{0}^{1,2}(\Omega)$. By Proposition 3.12 $\lambda$ is essential for $\left(f_{0}+I_{M}\right)$.

By Proposition 4.5 we can apply Theorem 3.20. Since $\left\{u_{\rho} / \rho: 0<\rho \leq\right.$ $\left.\rho_{0}\right\}$ is bounded in $L^{2}(\Omega)$ and

$$
\lim _{\rho \rightarrow 0^{+}} f\left(u_{\rho}\right) / \rho^{2}=\lambda,
$$

by (4.6) we deduce that $\left\{u_{\rho} / \rho: 0<\rho \leq \rho_{0}\right\}$ is bounded also in $W_{0}^{1,2}(\Omega)$. In particular

$$
\lim _{\rho \rightarrow 0^{+}} u_{\rho}=0 \text { in } W_{0}^{1,2}(\Omega) .
$$

Moreover, as in the proof of Proposition 4. $5_{\mathrm{ii}}$ we have

$$
\lim _{\rho \rightarrow 0^{+}} \rho^{-2} \int_{\Omega} \widetilde{G}\left(x, u_{\rho}\right) d x=\lim _{\rho \rightarrow 0^{+}} \rho^{-2} \int_{\Omega} \widetilde{G}\left(x, \rho\left(u_{\rho} / \rho\right)\right) d x=0,
$$

so that

$$
\lim _{\rho \rightarrow 0^{+}} \frac{1}{2 \rho^{2}} \int_{\Omega}\left(\sum_{i, j} a_{i j} D_{i} u_{\rho} D_{j} u_{\rho}+a_{0} u_{\rho}^{2}\right) d x=\lambda .
$$

Finally, by Lemma 4.12

$$
\lim _{\rho \rightarrow 0^{+}} u_{\rho}=0 \text { in } L^{\infty}(\Omega)
$$

so that $\left(\lambda_{\rho}, u_{\rho}\right)$ satisfies $(4.1)$ eventually as $\rho \rightarrow 0^{+}$. 


\section{Degiovanni}

We do not know whether all eigenvalues of (4.7) are essential. Similarly, we do not know whether all eigenvalues of (4.7) are of bifurcation for (4.1).

Now we shall consider two particular situations in which the condition that the eigenvalue is essential is not explicitely required.

The first one concerns the bifurcation from the first eigenvalue. If the set $\mathbf{K}$ is a cone (that is $\varphi_{1}$ takes only the values $-\infty, 0$ and $\varphi_{2}$ takes only the values $0,+\infty)$ and the nonlinearity $g$ has a more controlled growth, the problem has already been treated in [37]. On the other hand, for a fourth order variational inequality, the problem has been treated in [29], provided that $\varphi_{1}$ and $\varphi_{2}$ satisfy a further condition.

TIIEOREM 4.14. - Let us assume that $\varphi_{1}$ and $\varphi_{2}$ are not both zero quasieverywhere. Then

$$
\lambda=\inf \left\{\frac{1}{2} \int_{\Omega}\left(\sum_{i, j} a_{i j} D_{i} u D_{j} u+a_{0} u^{2}\right) d x: u \in \mathbf{K}_{0}, \frac{1}{2} \int_{\Omega} u^{2} d x=1\right\}
$$

is achieved, $\lambda$ is an eigenvalue of (4.7) (the first eigenvalue) and $\lambda$ is of bifurcation for (4.1). More precisely, all the thesis of Theorem 4.13 holds.

Proof.-By Proposition 4.9 we have $\mathbf{K}_{0} \neq\{0\}$. Therefore it is sufficient to apply Theorem 3.21 arguing as in the proof of Theorem 4.13.

In the second situation, concerning the case in which $f_{0}$ behaves like a quadratic form, we can give a bifurcation result also for higher eigenvalues.

Proposition 4.15. - Let us assume that the set

$$
\left\{x \in \Omega: \varphi_{1}(x)=0, \varphi_{2}(x)>0\right\} \cup\left\{x \in \Omega: \varphi_{1}(x)<0, \varphi_{2}(x)=0\right\}
$$

has null capacity. Then every eigenvalue of (4.7) is essential. Moreover, for every $\lambda$ in $\mathbf{R}$ the set $\left\{u \in \mathbf{K}_{0}:(\lambda, u)\right.$ satisfies (4.7) $\}$ is a linear subspace of $W_{0}^{1,2}(\Omega)$ of finite dimension.

Proof.-By Proposition $4.9 \mathrm{~K}_{0}$ is a linear subspace of $H$, so that

$$
\forall u, v \in H, f_{0}(u+v)+f_{0}(u-v)=2 f_{0}(u)+2 f_{0}(v) .
$$

If we apply Theorem 3.26 with $L$ equal to the identity map, the thesis follows. 
Bifurcation problems for nonlinear elliptic variational inequalities

THEOREM 4.16. - Let us assume that the set

$\left\{x \in \Omega: \varphi_{1}(x)=0, \varphi_{2}(x)>0\right\} \cup\left\{x \in \Omega: \varphi_{1}(x)<0, \varphi_{2}(x)=0\right\}$ has null capacity. Then every eigenvalue $\lambda$ of (4.7) is of bifurcation for (4.1). More precisely, all the thesis of Theorem 4.13 holds.

Proof. - It is sufficient to apply Theorem 3.29 .

Remark 4.17. - Let us suppose that there exists an open subset $\Omega^{\prime}$ of $\Omega$ such that

$$
\begin{gathered}
\varphi_{1}=\varphi_{2}=0 \text { quasi-everywhere in } \Omega / \Omega^{\prime} ; \\
\varphi_{1}<0 \text { and } \varphi_{2}>0 \text { quasi-everywhere in } \Omega^{\prime} .
\end{gathered}
$$

Then the assumption of Theorem 4.16 is satisfied and the eigenvalues of (4.7) are exactly the eigenvalues of the linear problem

$$
\left\{\begin{array}{l}
(\lambda, u) \in \mathbf{R} \times W_{0}^{1,2}\left(\Omega^{\prime}\right) \\
-\sum_{i, j} D_{i}\left(a_{i j} D_{j} u\right)+a_{0} u=\lambda u
\end{array} .\right.
$$

Finally we give a multiplicity result for simple eigenvalues. We point out that much more is known for equations (see $[5,6,7,21,26,28,34,35]$ ).

THEOREM 4.18. - Let us assume that the set

$$
\left\{x \in \Omega: \varphi_{1}(x)=0, \varphi_{2}(x)>0\right\} \cup\left\{x \in \Omega: \varphi_{1}(x)<0, \varphi_{2}(x)=0\right\}
$$

has null capacity. Let $\lambda$ be an eigenvalue of (4.7) such that $\left\{u \in \mathbf{K}_{0}:(\lambda, u)\right.$ satisfies (4.7)\} has dimension one and let $(\lambda, e)$ be a solution of (4.7) with $\frac{1}{2} \int_{\Omega} e^{2} d x=1$. Then there exist $\rho_{0}>0$,

$$
\begin{aligned}
& \left\{\lambda_{\rho}^{(h)}: 0<\rho \leq \rho_{0}, h=1,2\right\} \subset \mathbf{R} \\
& \left\{u_{\rho}^{(h)}: 0<\rho \leq \rho_{0}, h=1,2\right\} \subset \mathbf{K}
\end{aligned}
$$

such that $\left(\lambda_{\rho}^{(h)}, u_{\rho}^{(h)}\right)$ satisfies (4.1), $\frac{1}{2} \int_{\Omega}\left|u_{\rho}^{(h)}\right|^{2} d x=\rho^{2}$ and

$$
\begin{gathered}
\lim _{\rho \rightarrow 0^{+}} u_{\rho}^{(h)}=0 \text { in } L^{\infty}(\Omega) \text { and in } W_{0}^{1,2}(\Omega), \\
\lim _{\rho \rightarrow 0^{+}} \lambda_{\rho}^{(h)}= \\
=\lim _{\rho \rightarrow 0^{+}} \frac{1}{2 \rho^{2}} \int_{\Omega}\left(\sum_{i, j} a_{i j} D_{i} u_{\rho}^{(h)} D_{j} u_{\rho}^{(h)}+a_{0}\left|u_{\rho}^{(h)}\right|^{2}\right) d x=\lambda, \\
\lim _{\rho \rightarrow 0^{+}} u_{\rho}^{(1)} / \rho=e, \lim _{\rho \rightarrow 0^{+}} u_{\rho}^{(2)} / \rho=-e \text { in } W_{0}^{1,2}(\Omega) .
\end{gathered}
$$




\section{Degiovanni}

Proof. - Let us apply Theorem 3.30. We have only to remark that Theorem 3.30 gives

$$
\lim _{\rho \rightarrow 0^{+}} u_{\rho}^{(1)} / \rho=e, \lim _{\rho \rightarrow 0^{+}} u_{\rho}^{(2)} / \rho=-e
$$

in $L^{2}(\Omega)$.

On the other hand by Remark 3.10

$$
\frac{1}{2} \int_{\Omega}\left(\sum_{i, j} a_{i j} D_{i} e D_{j} e+a_{0} e^{2}\right) d x=\lambda
$$

and, as in the proof of Theorem 4.13,

$$
\lim _{\rho \rightarrow 0 \text { raise2pt }} \frac{1}{2 \rho^{2}} \int_{\Omega}\left(\sum_{i, j} a_{i j} D_{i} u_{\rho}^{(h)} D_{j} u_{\rho}^{(h)}+a_{0}\left|u_{\rho}^{(h)}\right|^{2}\right) d x=\lambda .
$$

Therefore the convergence holds also in $W_{0}^{1,2}(\Omega)$.

\section{Références}

[1] AттоuCH (H.). - Variational convergence for functions and operators, Applicable Mathematics Series, Pitman (Advanced Publishing Program), Boston, Mass. London, 1984 .

[2] Attouch (H.), Picard (C.). - Problèmes variationnels et théorie du potentiel non linéaire, Ann. Fac. Sci. Toulouse 1, 1979, p. 89-136.

[3] BENCI (V.). - Positive solutions of some eigenvalue problems in the theory of variational inequalities, J. Math. Anal. Appl. 61, 1977, p. 165-187.

[4] Benci (V.), Micheletti (A.M.). - Su un problema di autovalori per disequazioni variazionali, Ann. Mat. Pura Appl. (4) 107, 1975, p. 359-371.

[5] Berger (M.S.). - On von Karman's equations and the buckling of a thin elastic plate, I the clamped plate, Comm. Pure Appl. Math. 20 1967, p. 687-719.

[6] Berger (M.S.), Fife (P.C.). - Von Karman's equations and the buckling of a thin elastic plate, II plate with general edge conditions, Comm. Pure Appl. Math. 21, 1968, p. 227-241.

[7] Bонме (R.). - Die Lösung der Verzweigungsgleichungen für nichtlineare Eigenwertprobleme, Math. Z. 127, 1972, p. 105-126.

[8] Gobanov (G.), Marino (A.), Scolozzi (D.). - Evolution equation for the eigenvalue problem for the Laplace operator with respect to an obstacle (preprint), Dip. Mat. Pisa, 214, Pisa, 1987 .

[9] Gobanov (G.), Marino (A), Scolozzi (D.). - Multiplicity of eigenvalues for the Laplace operator with respect to an obstacle and non-tangency conditions. Nonlinear Anal., in press. 
Bifurcation problems for nonlinear elliptic variational inequalities

[10] DAL MASO (G.). - On the integral representation of certain local functionals, Ricerche Mat. 32, 1983, p. 85-113.

[11] De Giorgi (E.), Degiovanni (M.), Marino (A), Tosques (M.). - Evolution equations for a elass of nonlinear operators, Atti Accad. Naz. Lincei Rend. Cl. Sci. Fis. Mat. Natur. (8) 75, $198_{3}, 1-8198_{4}$.

[12] De Giorgi (E.), Franzoni (T.).- Su un tripo di convergenza variazionale, Rend. Sem. Mat. Brescia 3, 1979, p. 63-101.

[13] Degiovanni (M.).- Homotopical properties of a class of nonsmooth functions, Ann. Mat. Pura Appl., in press.

[14] Degiovanni (M.). - On the buckling of a thin elastic plate subjected to unilatera constraints, Nonlinear Variational Problems, (Isola d'Elba, 1986), Procceedings, in press.

[15] Degiovanni (M.), Marino (A.).- Alcuni problemi di autovalori e di biforcazione rispetto ad un ostacolo, Equazioni Differenziali e Calcolo delle Variazioni (Pisa, 1985), p. 71-93, Editrice Tecnico Scientifica, Pisa, 1985.

[16] Degiovanni (M.), Marino (A.). - Bifurcation for some nonlinear elliptic variational inequalities, Variational Methods in Differential Problems (Trieste, 1985), Rend. Istit. Mat. Univ. Trieste 18 1986, p. 40-48.

[17] Degiovanni (M.), Marino (A.). - Non-smooth variational bifurcation, Atti Accad. Naz. Lincei Rend. Cl. Sci. Fis. Mat. Natur. (8) 81, 1987, p. 259-269, 1988.

[18] Degiovanni (M.), Marino (A.), Tosques (M.). - Evolution equations with lack of convexity, Nonlinear Anal. 9, 1985, p. 1401-1443.

[19] Do (C.). - Bifurcation theory for elastic plates subjected to unilateral conditions, J. Math. Anal. Appl. 60 1977, p. 435-448.

[20] Do (C.). - Nonlinear bifurcation problem and buckling of an elastic plate subjected to unilateral conditions in its plane, Contemporary Developments in Continuum Mechanics and Partial Differential Equations (Proc. Internat. Sympos., Inst. Mat., Univ. Fed. Rio de Janeiro, Rio de Janeiro, 1977, p. 112-134, North-Holland Math. Studies, 30, North-Holland, Amsterdam, 1978.

[21] FADELL (E.R.), RABINOwitz (P.H.). - Bifurcation for odd potential operators and an alternative topological index, J. Funct. Anal. 26, 1977, p. 48-67.

[22] Krasnoselskir (M.A.). - Topological methods in the theory of nonlinear integral equations, Gosudarstv. Izdat. Techn. - Teor. Lit., Moscow, 1956. The Macmillan Co., New-York, 1964 .

[23] Kucera (M.). - A new method for obtaining eigenvalues of variational inequalities : operators with multiple eigenvalues, Czechoslovak Math. J. 32, 1982, p. 197-207.

[24] KuCERA (M.). - Bifurcation points of variational inequalities, Czechoslovak Math. J. 32, 1982, p. 208-226.

[25] Kucera (M.), Necas (J.), Soucek (J.). - The eigenvalue problem for variational inequalities and a new version of the Ljusternik-Schnirelmann theory, Nonlinear Analysis (Collection of papers in honor of Erich H. ROTHE), p. 125-143, Academic Press, New-York, 1978.

[26] Marino (A). - La biforcazione nel caso variazionale, Confer. Sem. Mat. Univ. Bari N. 132, 1973 . 


\section{Degiovanni}

[27] Marino (A), Scolozzi (D.).- Goedetiche con ostacolo, Boll. Un. Mat. Ital. B (6) 2,1983 , p. 1-31.

[28] McLEod (J.B), TuRner (R.E.L.). - Bifurcation for non-differentiable operators with an application to elasticity, Arch. Rational Mech. Anal. 63, 1976, p. 1-45.

[29] MiersemanN (E.). - Eigenwertaufgaben für Variation sungleichungen, Math. Nachr. 100, 1981, p. 221-228.

[30] Miersemann (E.).- - On higher eigenvalues of variational inequalities, Comment. Math. Univ. Carolin. 24, 1983 , p. 657-665.

[31] QUitTner (P.). - Spectral analysis of variational inequalities, Comment. Math. Univ. Carolin. 27, 1986 , p. 605-629.

[32] QuitTner (P.). - Bifurcation points and eigenvalues of inequalites of reactiondiffusion type, J. Reine Angew. Math. 380, 1987, p. 1-13.

[33] QUITTNER (P.). - Solvability and multiplicity results for variational inequalities, preprint.

[34] Rabinowitz (P.H.). - A bifurcation theorem for potential operators, J. Funct. Anal. 25, 1977 , p. 412-424.

[35] Rabinowitz (P.H.). - Minimax methods in critical point theory with applications to differential equations CBMS Regional Conference Series in Mathematics, 65, American Mathematical Society, Providence, R.I., 1986.

[36] Riddell (R.C.). - Eigenvalue problems for nonlinear elliptic variational inequalities on a cone, J. Funct. Anal. 26, 1977, p. 333-355.

[37] RIDdell (R.C.). - Eigenvalue problems for nonlinear elliptic variational inequalities, Nonlinear Anal. 3, 1979, p. 1-33.

[38] Spanier (E.H.). - Algebraic topology, McGraw, Hill Book Co., New-York, Toronto, Ont., London, 1966.

[39] Stampacchia (G.). - Le problème de Dirichlet pour les équations elliptiques du second ordre à coefficients discontinus, Ann. Inst. Fourier (Grenoble) 15, 1965, p. 189-257.

[40] SzULKIN (A.). - On a class of variational inequalities involving gradient operators, J. Math. Anal. Appl. 100, 1984, p. 486-499.

[41] SzULKIN (A.). - On the solvability of a class of semilinear variational inequalities, Rend. Mat. (7) 4, 1984 , p. 121-137.

[42] SzULKIN (A.).- - Positive solutions of variational inequalities : a degree-theoretic approach, J. Differential Equations 57, 1985, p. 90-111. 